\title{
WestVirginiaUniversity
}

THE RESEARCH REPOSITORY @ WVU

Graduate Theses, Dissertations, and Problem Reports

2016

\section{Echo Phenomena in Populations of Chemical Oscillators}

\author{
Tianran Chen
}

Follow this and additional works at: https://researchrepository.wvu.edu/etd

\section{Recommended Citation}

Chen, Tianran, "Echo Phenomena in Populations of Chemical Oscillators" (2016). Graduate Theses, Dissertations, and Problem Reports. 5350.

https://researchrepository.wvu.edu/etd/5350

This Thesis is protected by copyright and/or related rights. It has been brought to you by the The Research Repository @ WVU with permission from the rights-holder(s). You are free to use this Thesis in any way that is permitted by the copyright and related rights legislation that applies to your use. For other uses you must obtain permission from the rights-holder(s) directly, unless additional rights are indicated by a Creative Commons license in the record and/ or on the work itself. This Thesis has been accepted for inclusion in WVU Graduate Theses, Dissertations, and Problem Reports collection by an authorized administrator of The Research Repository @ WVU. For more information, please contact researchrepository@mail.wvu.edu. 


\section{Abstract}

\section{Echo Phenomena in Populations of Chemical Oscillators \\ Tianran Chen}

The emergence of collective behavior has been observed in all levels of biological systems, for example, the aggregation of slime mold, swarm motion of insects and the collective motion in schools of fish. Synchronization is one of the most important collective behaviors and can play a pivotal role in maintaining the normal function of a living system, such as pacemaker cells in the heart, circadian rhythms, and insulin release from pancreatic cells. Synchronization typically arises as a result of the interaction of large ensembles of oscillators. Studies of the Belousov-Zhabotinsky (BZ) chemical oscillators have shown a variety of collective dynamical behaviors, such as phase clusters, dynamical quorum sensing and chimera states. The discovery of echo phenomena in large populations of coupled Kuramoto oscillators motivates us to study this dynamical behavior using photosensitive BZ oscillators.

In this thesis, we examine echo behavior experimentally and mathematically. The experiments are carried out with a BZ micro-oscillator system. A large system of micro-oscillators is achieved by the design of a large oscillator array (LOA), which permits coupling of over 1000 oscillators. The dimensionless Zhabotinsky, Buchholtz, Kiyatkin and Epstein (ZBKE) mathematical model is used to investigate the behavior. The experiment and numerical results illustrate that if a BZ system of oscillators is subject to two perturbations, separated by time $\tau$, then at the time $\tau$ after second perturbation, the oscillators show a measurable response in their collective signal. Factors such as noise and size of the perturbation impacting the magnitude of the echo are examined and a theoretical calculation of the magnitude of the echo as a function of the size of the perturbation exhibits good agreement with simulation results using the ZBKE model. 


\section{Acknowledgments}

I am sincerely grateful to my advisor, Dr. Kenneth Showalter, for the continuous support of my graduate research and study, for his great patience, incredible enthusiasm and wealth of knowledge to make my research project possible. I would like to give my spe-

cial thanks to Dr. Mark Tinsley for his fantastic research insights, mathematical modeling skills, continuous guidance with my research and great personality. I also want to give my thanks to my committee members: Dr. Fabien Goulay, Dr. Justin Legleiter and Dr. Mark Tinsley, for taking the time to evaluate my thesis. Thank you to all my labmates in WVU nonlinear chemical dynamics: Dr. Simbarashe Nkomo, Dr. Darrell Collision, Dr. Razan Snari, Desmond Yengi, Sadegh Faramarzi and Jaz Jamaluddin for their kind help and insightful discussions during my graduate study. Thank you also to Allen Burns for building the experimental setup.

I am thankful to all my teachers for their instruction and inspiration during my school education, and also my friends in the U.S. and China who always have been there for me.

Last, I would like to express my deepest gratitude to my family: my father Chen Weiguo, my mother Liang Lihua, my grandparents and other relatives for support and encouragement throughout my life. Special gratitude goes to my fiancée Ju Lin for continuous support and motivation with infinite love. 


\section{Contents}

Contents $\quad$ iv

List of Figures $\quad$ vi

List of Tables

1 Collective Behavior of Oscillators $\quad 1$

1.1 Oscillation of Pendulum . . . . . . . . . . . . . . . . . 1

1.2 Synchronization of Coupled Oscillators . . . . . . . . . . . . . 3

1.3 Collective Behaviors of Coupled Oscillators . . . . . . . . . . . . . 6

Bibliography 11

2 The Belousov-Zhabotinsky Reaction $\quad 16$

2.1 The Belousov-Zhabotinsky Reaction . . . . . . . . . . . . . 16

2.2 The FKN Mechanism of the BZ Reaction . . . . . . . . . . . . . . 17

2.3 ZBKE Model . . . . . . . . . . . . . . . . . . . . . . . . . . 18 
2.4 The Photosensitive BZ Reaction and Modified ZBKE Model . . . . . . . . . 22

2.5 Experimental Setup . . . . . . . . . . . . . . . . . . 25

2.6 Phase Response Curve . . . . . . . . . . . . . . . . . . . . . . . . . . . . . 28

$\begin{array}{ll}\text { Bibliography } & 33\end{array}$

3 Echo Phenomena in Populations of Chemical Oscillators 37

3.1 Introduction . . . . . . . . . . . . . . . . . . . . 37

3.2 Experiment . . . . . . . . . . . . . . . . . . . . . 38

3.3 Simulation . . . . . . . . . . . . . . . . . . . . . 41

3.4 Geometric Approach . . . . . . . . . . . . . . . . . . . . . 54

3.5 Conclusion . . . . . . . . . . . . . . . . . . . . . 57

$\begin{array}{lr}\text { Bibliography } & 59\end{array}$ 


\section{List of Figures}

1.1 The osillcation of two pendulums with length $l=1.0$ (blue) and $l=0.2$ (green). 2

1.2 The phase of oscillator with $\theta=0.5$ in the unit circle . . . . . . . . . . 4

1.3 (a) In-phase synchronization of coupled Kuramoto oscillators. The blue oscillator has $\theta_{(0)}=0.0$ and $\omega=0.0$; the green oscillator has $\theta_{(0)}=3.2$ and $\omega=0.5$. The coupling constant $K=0.04$. (b) Order paramter of the coupled oscillators as function of time. . . . . . . . . . . . . . . . . .

1.4 Anti-phase synchronization of coupled Kuramoto oscillators. The blue oscillator has $\theta_{(0)}=0.0$ and $\omega=0.0$, and the green oscillator has $\theta_{(0)}=0.1$ and $\omega=0.5$. The coupling strength $K=-0.04 \ldots \ldots \ldots \ldots \ldots$

1.5 Numerical simulation of the 500 coupled Kuramoto oscillators: (a) coupling strength $=0.05 ;(\mathrm{b})$ coupling strength $=1.8 ;(\mathrm{c})$ coupling strength $=6.6 ;(\mathrm{d})$ order parameter as a function of the coupling strength, with values indicated by red dots for (a), (b) and (c); (e) natural frequency distribution of the 500 oscillators. 9 
2.1 (a) Experimental setup: charge-coupled device (CCD) camera records the dynamics of each individual oscillator. The image is processed by the computer and the feedback illumination is applied to the oscillators via a spatial light modulator (SLM). (b) The large oscillator array (LOA) is recorded by the CCD camera. . .

2.2 Time series showing transmitted light intensity $\ldots \ldots \ldots \ldots \ldots$

2.3 Experiment and simulation of PRCs under different sizes of simulus. (a) Experiments: (i) perturbation of high light intensity, $\phi=2.10 \mathrm{~mW} \mathrm{~cm}^{-2}$, blue; (ii) perturbation of low light intensity, $\phi=1.05 \mathrm{~mW} \mathrm{~cm}^{-2}$, red. (b) Simulations: (i) high intensity perturbation, $\phi=0.147$, blue; (ii) low intensity perturbation, $\phi=$

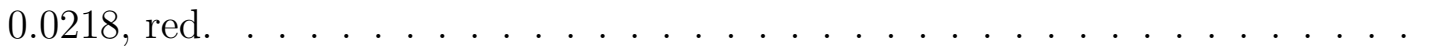

3.1 Experimental measurements illustrating echo behavior in populations of BelousovZhabotinsky (BZ) oscillators. The order parameter $R$ is calculated from the phases of the oscillators and plotted as a function of time. The phases of the oscillators are determined using linear interpolation between consecutive peak times. An echo is exhibited in the magnitude of the order parameter $R$ at time $\mathrm{t}_{p}+2 \tau$ for a system perturbed at times $t_{p}$ and $t_{p}+\tau$. (a) Uncoupled system, $k=$ 0 , with $t_{p}=376 \mathrm{~s}, \tau=378 \mathrm{~s}$ and $N=1295$. (b) Coupled system, $k=0.25$, with $t_{p}=373 \mathrm{~s}, \tau=336 \mathrm{~s}$ and $N=1001$. Average natural period and standard deviation in (a) $T_{0}=36.5 \pm 3.2 \mathrm{~s}$, (b) $T_{0}=42.6 \pm 8.2 \mathrm{~s} \ldots \ldots \ldots \ldots$ 
3.2 Plots of phase as a function of frequency of oscillators from the experiment shown in Figure 3.1(a) at the following times: (a) Immediately prior to first perturbation at $t_{p}$, (b) immediately following the first perturbation, in which the oscillators in the resetting region of their phase are phase reset to 0 , (c) half-way between the two perturbations, $t_{p}+\tau / 2$, (d) immediately prior to the second perturbation, (e) immediately following the second perturbation, in which oscillators in the resetting region of their phase are phase reset to 0 and (f) at the time of the echo. 42

3.3 Simulations of the modified ZBKE model illustrating echo behavior in populations of uncoupled photosensitive BZ oscillators. The order parameter $R$ is calculated from the phases of the oscillators and plotted as a function of time. An echo is exhibited in the magnitude of $R$ at time $t_{p}+2 \tau$ for a system perturbed at times $t_{p}$ and $t_{p}+\tau$. (a) System with $k=0, \mathrm{t}_{p}=500, \tau=800$, and $N=5000$. Inset, magnitude of the echo as a function of the noise intensity D. (b) System with $k$ $=0, t_{p}=5000, \tau=40000$, and $N=5000$. Average natural period $T_{0}=42.5 \pm$ 2.4 (dimensionless time units) . . . . . . . . . . . . . . . . . . . . . . 44

3.4 (a) Numerical simulation of a coupled system with $k=3.35 \times 10^{-4}, \tau=650$, and $N=5000$. Note the slower phase dispersion following each perturbation and the slightly larger magnitude of the echo. (b) Simulated maximum value of the order parameter at the time of the echo as a function of coupling strength. . . . . . 
3.5 Magnitude of the simulated order parameter $R$ at the time of the echo as a function of the size of the phase resetting region, $X$, which increases monotonically with the size of the perturbation. ZBKE simulation values (dark blue line), values calculated using groups I and II (light blue line), values calculated using groups I IV (red dashed line). (b) Simulated phase response curves for stimulus $\Phi_{\text {perturb }}=$ 0.147 (blue line) and $\Phi_{\text {perturb }}=0.0218$ (red line). The size of the associated phase resetting regions is shown by the red and blue horizontal arrows, respectively. Simulation parameters as in Figure $3.3 \ldots \ldots \ldots \ldots \ldots$

3.6 Plots of phase as a function of frequency of oscillators in the ZBKE simulation shown in Fig. 2(a) at the following times: (a) Immediately prior to first perturbation at $t_{p}$, (b) immediately following the first perturbation, in which oscillators in the resetting region of their phase are phase reset to $0,(\mathrm{c})$ half-way between the two perturbations, $t_{p}+\tau / 2$, (d) immediately prior to the second perturbation, (e) immediately following the second perturbation, in which oscillators in the phase range $X$ through $(1-X)$ are phase reset to 0 , and (f) at the time of the echo when a series of narrow bands, each with a single branch segment is observed. Parameters: $\tau=650$; other parameters as in Figure $3.3 . \ldots \ldots \ldots$

3.7 Phase as a function of the frequency of oscillators from ZBKE simulation for (a) group I oscillators and (b) group II oscillators at various times: (i) Immediately prior to the first perturbation, (ii) immediately following the second perturbation, and (iii) at the time of the echo. All parameters as in Figure $3.3 . \ldots \ldots \ldots$ 
3.8 (a) Predicted phase distribution of oscillators (solid lines) and ZBKE simulation values (symbols) at the time of the echo. Green, red, black and blue lines correspond to groups I, II, III and IV, respectively. The violet line corresponds to the sum of the four groups. The ZBKE simulations were carried out with a large number of oscillators, $N=100$, 000. (b) Detail of structure from Figure 3.6, color coded according to the four different groups in (a) at the time of the echo. The frequencies indicated by the left and right red circles correspond to $14 / \tau$ and

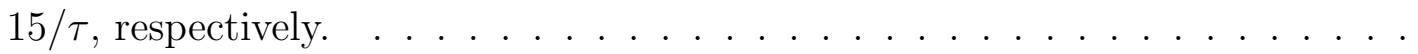

3.9 Geometric determination of phase distribution of group III oscillators. (a) At time $t_{p}$, they are uniformly distributed through the refractory region. (b) Just prior to the second perturbation, the same oscillators are now uniformly distributed through the excitable region. (c) The oscillators are then phase reset by the perturbation. The red, light blue and green regions indicate the oscillators in one of three narrow phase ranges at time $t_{p}$. The order of their vertical stacking in (b) and (c) is arbitrary. Each horizontal band equally contributes to the total number of oscillators at a particular phase. (d) The phase range of each set, red, blue and green, of oscillators at the time of the echo. Since the choice of $\epsilon$ was arbitrary, the phase spread of the other oscillators is bound by the sides of the parallelogram, black dotted line. $\ldots \ldots \ldots \ldots \ldots \ldots$ 


\section{List of Tables}

2.1 ZBKE model reaction scheme . . . . . . . . . . . . . . . . . . . . . 19

2.2 Scalings for the three variable ZBKE model . . . . . . . . . . . . . . . . 23 


\section{Chapter 1}

\section{Collective Behavior of Oscillators}

\subsection{Oscillation of Pendulum}

Oscillations are common phenomena that appear in daily life, such as the swinging of a pendulum clock, the blinking of a turn signal, the beating of the heart and the circadian rhythms of the body $[4,19,3]$. In an oscillatory system, the motion is periodic and is typically characterized by a certain frequency and amplitude. Figure 1.1 shows the oscillation of two ideal pendulums swinging with small amplitude. The oscillation is governed by the following equation:

$$
\frac{d^{2} \theta}{d t^{2}}+\frac{g}{l} \sin \theta=0
$$

where $\theta$ is the angular displacement, $l$ is the length of the pendulum and $g$ is the constant 


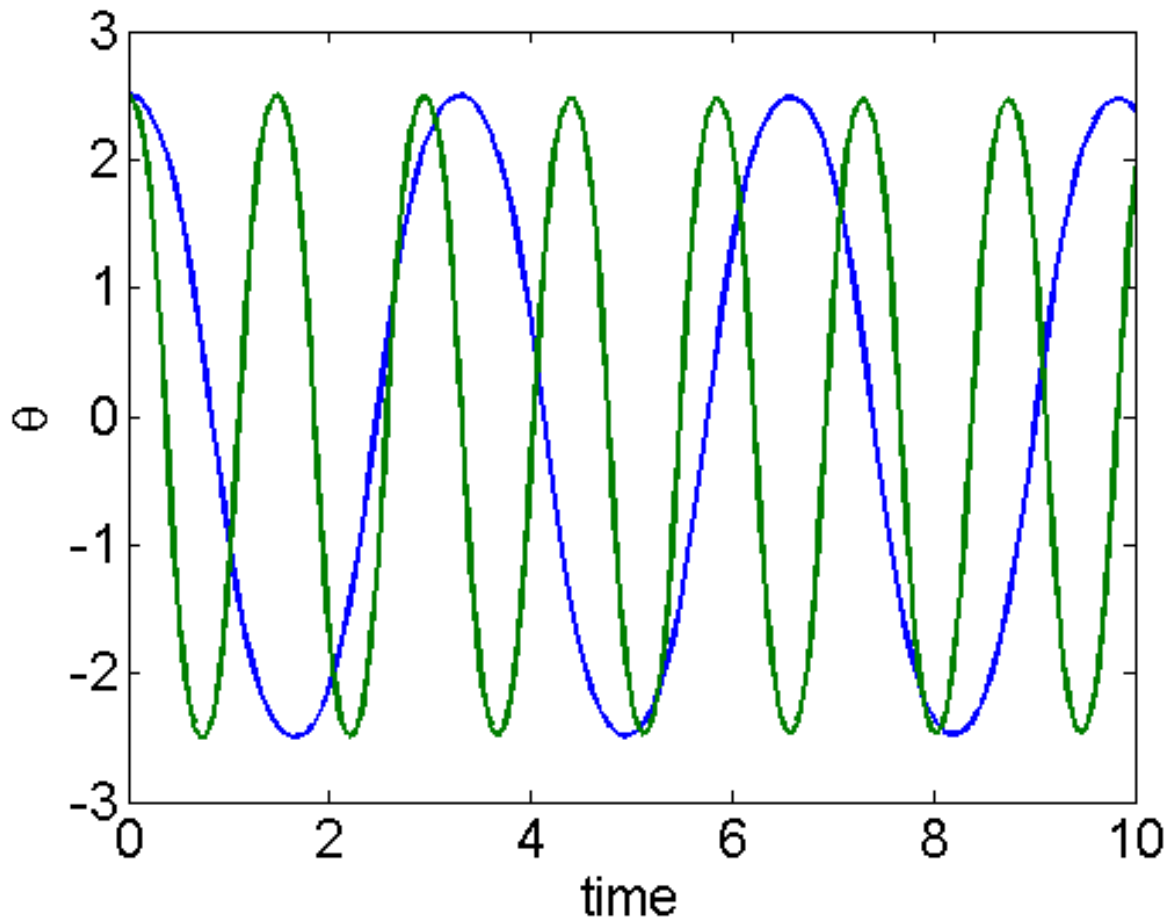

Figure 1.1: The osillcation of two pendulums with length $l=1.0$ (blue) and $l=0.2$ (green).

of gravitational acceleration. The amplitude, period and phase are the most important properties to describe any oscillation. The amplitudes of the two oscillators, blue and green, are $2.5^{\circ}$, which represents the maximum displacement from the equilibrium position $(\theta=$ $0^{\circ}$. The period is the time taken for one complete oscillation cycle. The two pendulums oscillate with different periods due to the differing lengths of the pendulums. The longer $l$ pendulum leads to a larger period (blue). The phase defines the specific state of a periodic oscillator and is important in characterizing synchronization, which will be discussed in next section. 


\subsection{Synchronization of Coupled Oscillators}

Synchronization is one of the most fascinating cooperative phenomenon appearing in bi-

ological $[5,6,32]$, mechanical $[12,16,20]$ and chemical oscillatory systems $[10,31,30]$. A classic example of synchronization in nature occurs in the community of Southeast Asian fireflies [22]. Hundreds of thousands of fireflies aggregate in trees along the river bank and flash in synchrony for hours during the night. A general mechanism behind different types of synchronization phenomena mystified scientists for years. In 1975, the Japanese physicist Yoshiki Kuramoto proposed a tractable mathematical model [13] to investigate synchronization phenomena. This mathematical model came to be known as the Kuramoto model. The typical form of the governing equations are given by [13]:

$$
\frac{d \theta_{i}}{d t}=\omega_{i}+\frac{K}{N} \sum_{j=1}^{N} \sin \left(\theta_{j}-\theta_{i}\right)
$$

where $N$ is number of oscillators, $\omega_{i}$ is the natural frequency of $i^{t h}$ oscillator and $K$ is coupling strength, which determines the strength of interaction between the coupled oscillators [2]. $\theta$ is the phase variable, which defines the specific state in given osciilatory cycle. The phase $\theta$ can be mapped into a unit circle (radius of 1), as shown in Figure 1.2. The filled red circle indicates the state of the oscillator at $\theta=0.5$. This unit circle is also called a phase circle. The phase circle is in the complex plane, in which the complex number $z$ associated with an oscillator is calculated by the following equation: 


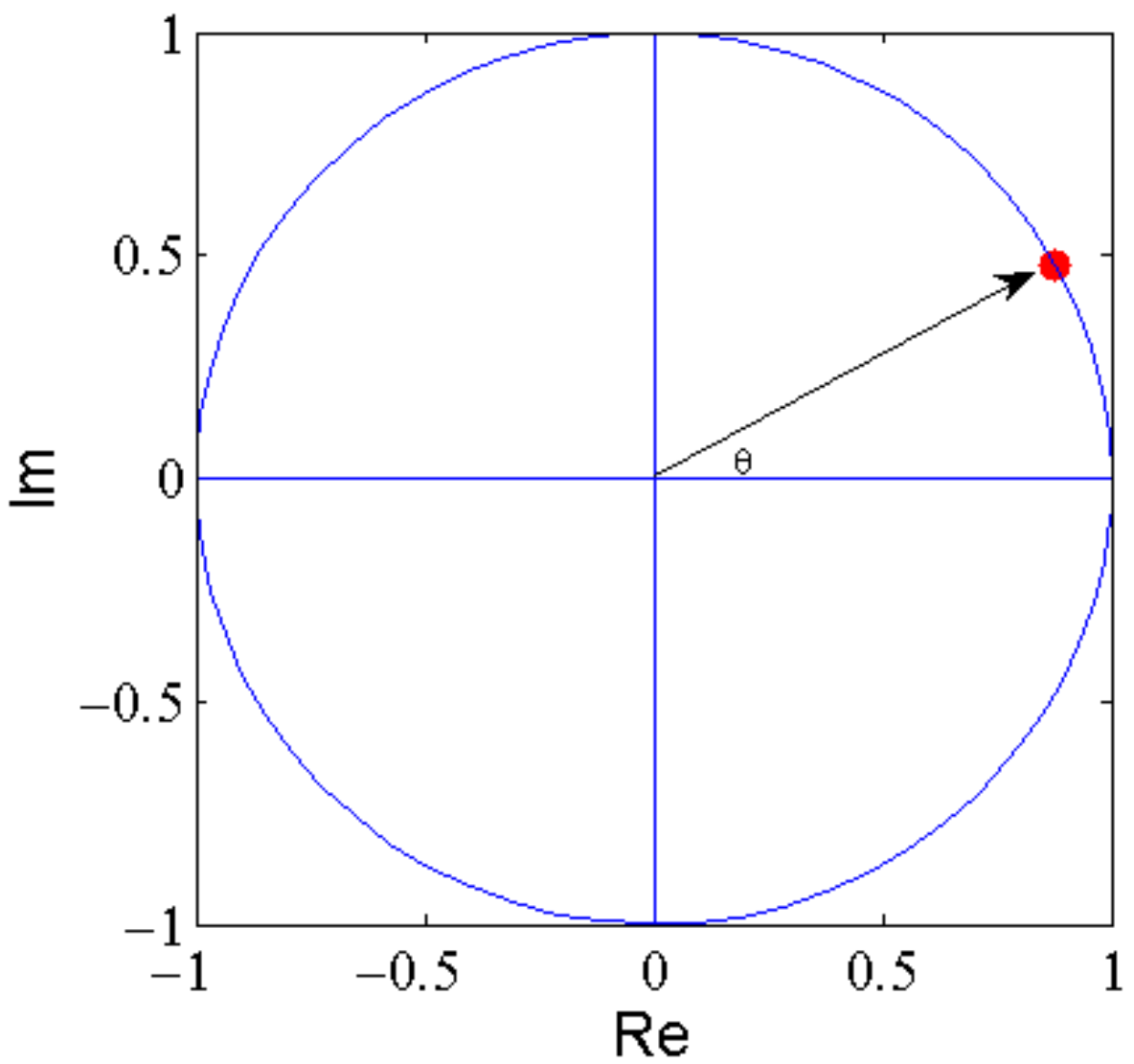

Figure 1.2: The phase of oscillator with $\theta=0.5$ in the unit circle

$$
z=e^{i \theta}
$$

where $i=\sqrt{-1}$ and $e \approx 2.718$ is Euler number.

The dynamics of Kuramoto oscillators are completely determined by their phases and this type of oscillator is therefore called a phase oscillator. A simple type of synchronization phenomenon can be demonstrated using two coupled Kuramoto oscillators, as shown in 
Figure 1.3(a). These two coupled, non-identical oscillators start with different phases and gradually synchronize to a common frequency due to their interaction, which is determined by the difference between their phases. Owing to the complete overlap of the phase of the two oscillators, this particular synchronization is known as in-phase synchronization. Another state of synchronization is known as anti-phase synchronization. This occurs when two coupled oscillators synchronize with a constant phase difference of $\pi$, as shown in Figure 1.4. The anti-phase synchronization can be achieved using a negative coupling strength between two phase oscillators.

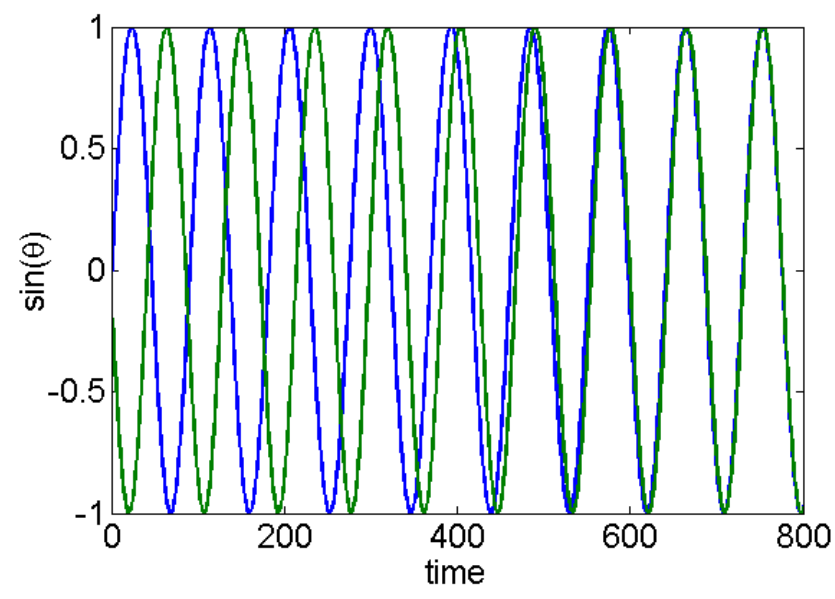

(a)

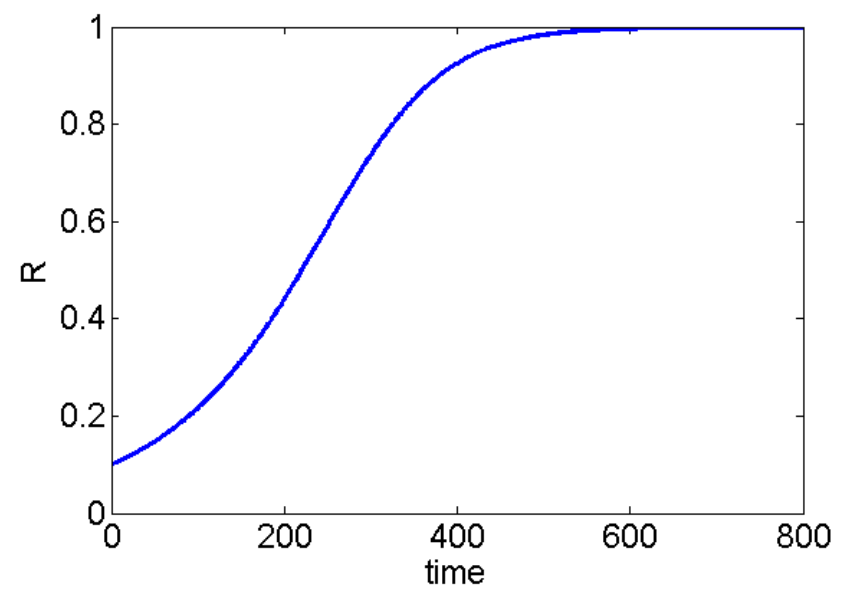

(b)

Figure 1.3: (a) In-phase synchronization of coupled Kuramoto oscillators. The blue oscillator has $\theta_{(0)}=0.0$ and $\omega=0.0$; the green oscillator has $\theta_{(0)}=3.2$ and $\omega=0.5$. The coupling constant $K=0.04$. (b) Order paramter of the coupled oscillators as function of time.

To quantify the degree of synchrony, Kuramoto introduced an order parameter $R$, which describes the phase-coherence of a community of oscillators. The order parameter is defined 
by the following equation $[13,2]$ :

$$
R e^{i \psi}=\frac{1}{N} \sum_{j=1}^{N} e^{i \theta_{j}},
$$

where $R$ is the order parameter, $\psi$ is the average phase, $N$ is the number of oscillators and $\theta_{j}$ is the phase of $j^{\text {th }}$ oscillator. The order parameter $R$ has values from $0 \leq \mathrm{R} \leq 1$. When $\mathrm{R} \approx 1$, the oscillators are completely synchronized, and when $R \approx 0$, the oscillators are completely asynchronous. Partial synchronization occurs when the value of $R$ is between these two boundaries. Figure 1.3(b) shows a plot of the order parameter versus time for the in-phase synchronization of two coupled Kuramoto oscillators. The order parameter starts with a small value $(\mathrm{R} \approx 0.1)$, which indicates almost no phase-coherence between these two oscillators. $R$ gradually approachs the value of one as the two coupled oscillators begin to synchronize. When time $\approx 650$, the phases and frequencies of the two coupled oscillators become identical.

\subsection{Collective Behaviors of Coupled Oscillators}

Rich and complex collective dynamical behaviors can arise from ensembles of interacting oscillators. The emergence of collective behavior depends on multiple factors, such as the nature of the oscillatory system, number of oscillators and the form of coupling. One of the most important factors is the strength of interaction, given by the coupling strength, 


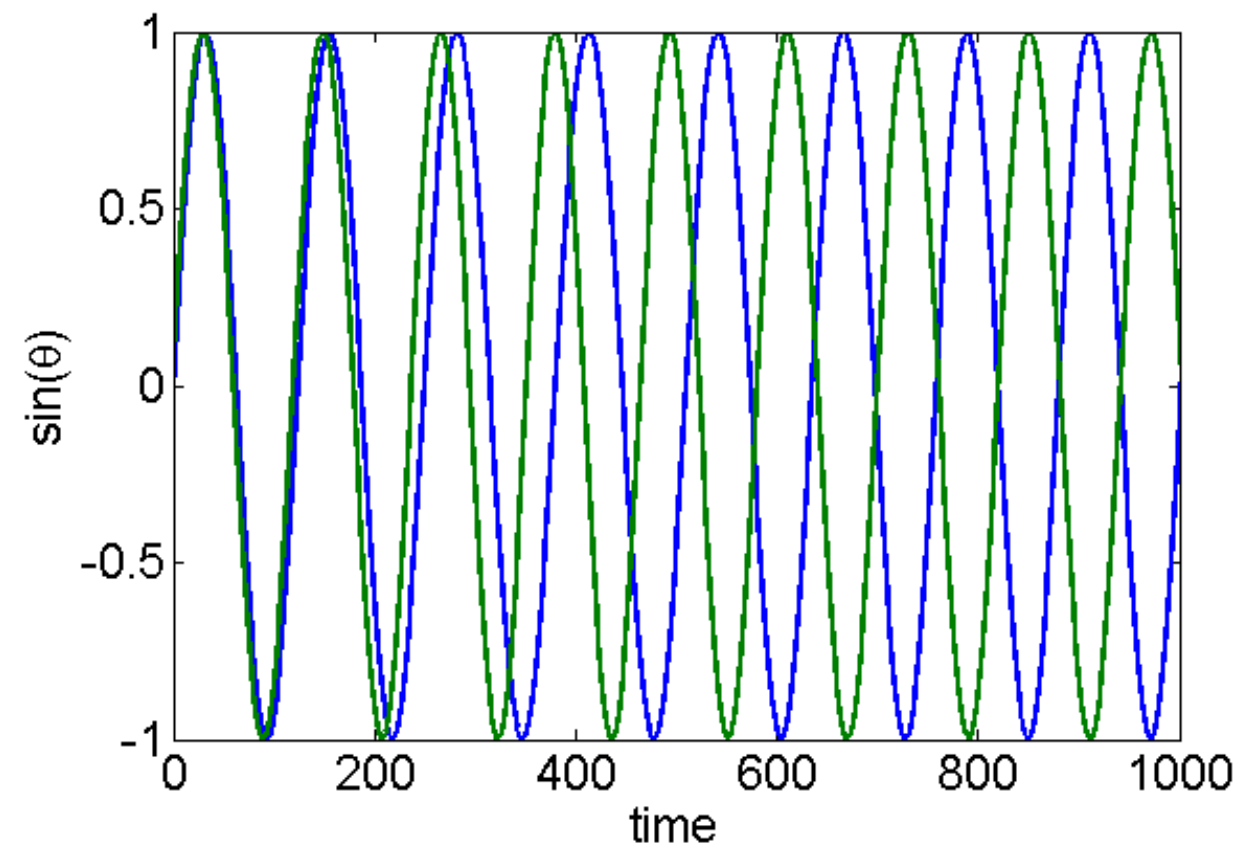

Figure 1.4: Anti-phase synchronization of coupled Kuramoto oscillators. The blue oscillator has $\theta_{(0)}=0.0$ and $\omega=0.0$, and the green oscillator has $\theta_{(0)}=0.1$ and $\omega=0.5$. The coupling strength $K=-0.04$.

$K$. In a large population of globally coupled Kuramoto oscillators, the degree of synchrony increases with increasing coupling strength; the oscillators gradually change from randomly distributed phase to a common frequency and phase. This type of synchronization behavior is referred to as Kuramoto synchronization [2]. The collective signal can be described by the order parameter. In Figure 1.5(d), the value of order parameter sigmoidally grows as the coupling strength increases. The simulation results are performed using globally coupled Kuramoto oscillators $(N=500)$. To visualize the state of each oscillator, their phases can be plotted on the phase circle. Three different magnitudes of $K, 0.05,1.8$ and 6.6 , are used, 
with the results shown in Figure 1.5(a), 1.5(b), 1.5(c). For clarity, only the first 200 of the 500 oscillators are plotted. The corresponding value of $R$ for each coupling strength $(K=$ $0.05,1.8,6.6)$ is indicated as a red dot in Figure $1.5(\mathrm{~d})$. With a coupling constant of $K$ $=0.05$ (weak coupling strength), the oscillators randomly distribute along the phase circle, showing an incoherent state. When $K$ is increased to 1.8 (medium coupling strength), the oscillators partially synchronize, in which some of them synchronize and the rest of them oscillate at their natural frequency. For $K=6.6$ (strong coupling strength), the oscillators completely in-phase synchronize. Figure 1.5(e) shows the natural frequency distribution of 500 oscillators. The frequencies are randomly selected from a Gaussian distribution.

In addition to complete in-phase synchronization, the populations of globally coupled oscillators can also evolve from an incoherent state to phase clusters. This behavior can occur during the transition before complete synchronization, in which the coupled oscillators spontaneously form multiple synchronized groups with a constant phase difference between the groups. Synchronized cluster phenomena have been observed theoretically with the Kuramoto model $[8,33]$ and experimentally in electrochemical oscillatory [11] and chemical oscillatory systems $[24,26]$.

Another type of transition, as well as the Kuramoto transition, has been observed in biological systems. It is known as a quorum sensing transition. Collective behaviors of bacteria, such as biofilm formation, virulence and antibiotic resistance $[18,23,7]$ are thought to be regulated and coordinated by quorum sensing type transitions. The bacteria produce and release signaling molecules, called autoinducers, in which the concentration of the autoinducer 


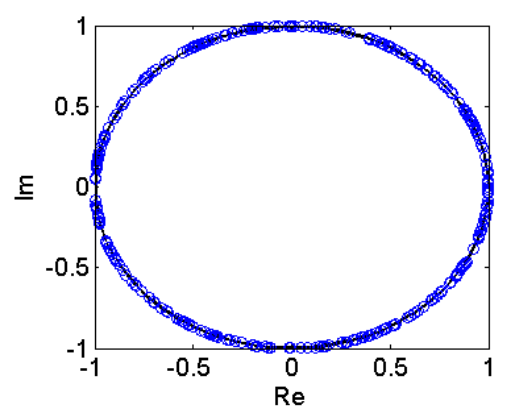

(a)

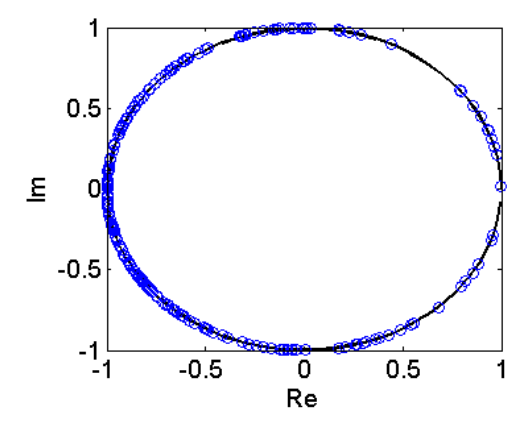

(b)

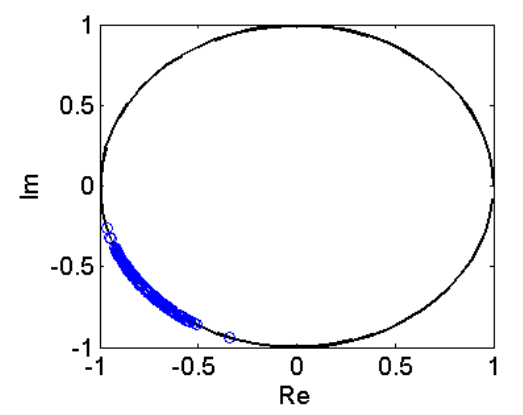

(c)

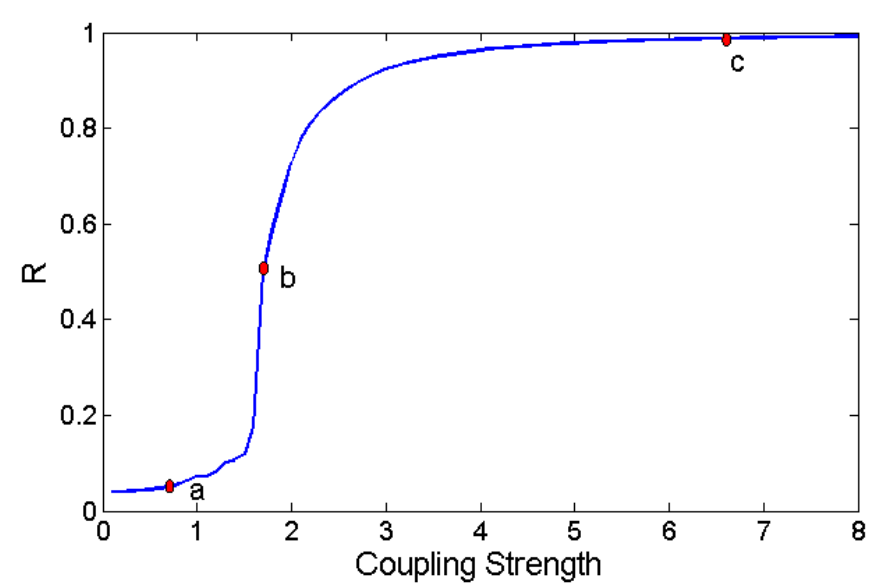

(d)

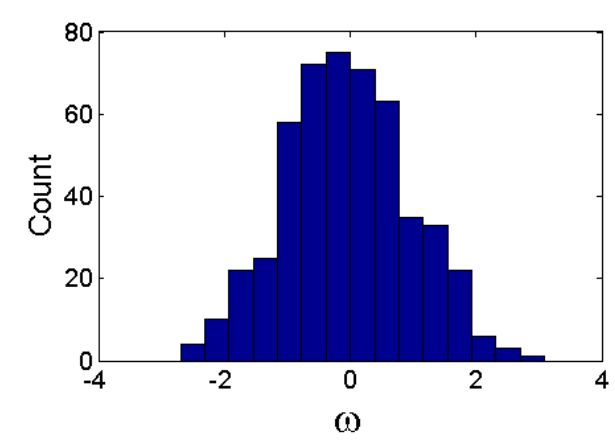

(e)

Figure 1.5: Numerical simulation of the 500 coupled Kuramoto oscillators: (a) coupling strength $=0.05 ;(\mathrm{b})$ coupling strength $=1.8 ;(\mathrm{c})$ coupling strength $=6.6 ;(\mathrm{d})$ order parameter as a function of the coupling strength, with values indicated by red dots for (a), (b) and (c); (e) natural frequency distribution of the 500 oscillators. 
molecule corresponds to the population density of the bacteria [15]. Collective behaviors of various types will arise once the concentration of the signaling molecule reaches a certain threshold. Quorum sensing transition phenomena also have been observed using local or global coupled chemical oscillators $[29,28,25]$. Another peculiar collective behavior of coupled oscillators consists of incongruous behaviors of groups: one coherent and synchronized, the other incoherent and asynchronous. This phenomenon is known as the chimera state [1]. It was discovered and studied using the Kuramoto model $[14,21]$. The chimera state has been investigated experimentally using coupled chemical oscillators [27] and coupled-map lattices [9].

Recently, E. Ott et al. [17] reported a remarkable collective behavior using a population of weakly coupled Kuramoto oscillators, referred to as echo phenomena. This thesis provides insight into echo phenomena using heterogeneous photosensitive Belousov-Zhabotinsky (BZ) oscillators. The detailed reaction mechanism and mathematical model of the BZ reaction are described in Chapter 2. In Chapter 3, echo phenomena are studied with large populations of coupled and uncoupled oscillators. The experimental behavior is consistent with the behavior of a mathematical model. 


\section{Bibliography}

[1] D. M. Abrams and S. H. Strogatz. "Chimera states for coupled oscillators". Phys. Rev. Lett., 93(17):174102, 2004.

[2] J. A. Acebrón, L. L. Bonilla, C. J. P. Vicente, F. Ritort, and R. Spigler. "The Kuramoto model: A simple paradigm for synchronization phenomena". Rev. Mod. Phys., 77(1):137-185, 2005.

[3] J. Aschoff and R. Wever. "Human circadian rhythms: A multioscillatory system". Fed. Proc., 35(12):236-232, 1976.

[4] A. Balanov, N. Janson, D. Postnov, and O. Sosnovtseva. Synchronization: From Simple to Complex. Springer Science \& Business Media, 2008.

[5] J. Buck. "Synchronous rhythmic flashing of fireflies". The Q. Rev. Biol., 13(3):301-314, 1938.

[6] J. Buck and E. Buck. "Mechanism of rhythmic synchronous flashing of fireflies: Fireflies of Southeast Asia may use anticipatory time-measuring in synchronizing their flashing". 
Sci., $\mathbf{1 5 9}(3821): 1319-1327,1968$.

[7] J. W. Costerton, P. S. Stewart, and E. P. Greenberg. "Bacterial biofilms: A common cause of persistent infections". Sci., 284(5418):1318-1322, 1999.

[8] D. Golomb, D. Hansel, B. Shraiman, and H. Sompolinsky. "Clustering in globally coupled phase oscillators". Phys. Rev. A, 45(6):3516-3530, 1992.

[9] A. M. Hagerstrom, T. E. Murphy, R. Roy, P. Hövel, I. Omelchenko, and E. Schöll. "Experimental observation of chimeras in coupled-map lattices". Nat. Phys., 8(9):658$661,2012$.

[10] V. Horvath, P. L. Gentili, V. K. Vanag, and I. R. Epstein. "Pulse-coupled chemical oscillators with time delay". Angewandte Chemie Int. Ed., 51(28):6878-6881, 2012.

[11] I. Z. Kiss, Y. Zhai, and J. L. Hudson. "Characteristics of cluster formation in a population of globally coupled electrochemical oscillators: An experiment-based phase model approach". Prog. Theor. Phys. Suppl., 161(161):99-106, 2006.

[12] E. Klarreich. "Discovery of coupled oscillation put 17th-century scientist ahead of his time". SIAM News, 35(8):1-3, 2002.

[13] Y. Kuramoto. Chemical Oscillations, Waves, and Turbulence. Springer Science \& Business Media, 2012. 
[14] Y. Kuramoto and D. Battogtokh. "Coexistence of coherence and incoherence in nonlocally coupled phase oscillators". Nonlinear Phenom. Complex Syst., 5(4):380-385, 2002.

[15] M. B. Miller and B. L. Bassler. "Quorum sensing in bacteria". Annu. Rev. Microbiol., 55:165-199, 2001.

[16] G. V. Osipov, J. Kurths, and C. Zhou. Synchronization in Oscillatory Networks. Springer Science \& Business Media, 2007.

[17] E. Ott, J. H. Platig, T. M. Antonsen, and M. Girvan. "Echo phenomena in large systems of coupled oscillators". Chaos, 18(3):037115, 2008.

[18] G. M. Patriquin, E. Banin, C. Gilmour, R. Tuchman, E. P. Greenberg, and K. Poole. "Influence of quorum sensing and iron on twitching motility and biofilm formation in Pseudomonas aeruginosa". J. Bacteriol., 190(2):662-671, 2008.

[19] A. Pikovsky, M. Rosenblum, and J. Kurths. Synchronization: A Universal Concept in Nonlinear Sciences. Cambridge university press, 2003.

[20] J. W. S. B. Rayleigh. "The theory of sound". Nat., 58:121-122, 1898.

[21] S. Shima and Y. Kuramoto. "Rotating spiral waves with phase-randomized core in nonlocally coupled oscillators". Phys. Rev. E, 69(3):036213, 2004.

[22] H. M. Smith. "Synchronous flashing of fireflies". Sci., 82(2120):151-152, 1935. 
[23] P. S. Stewart and J. W. Costerton. "Antibiotic resistance of bacteria in biofilms.". The Lancet, 358(9276):135-138, 2001.

[24] A. F. Taylor, P. Kapetanopoulos, B. J. Whitaker, R. Toth, L. Bull, and M. R. Tinsley. "Clusters and switchers in globally coupled photochemical oscillators". Phys. Rev. Lett., 100(21):214101, 2008.

[25] A. F. Taylor, M. R. Tinsley, F. Wang, Z. Huang, and K. Showalter. "Dynamical quorum sensing and synchronization in large populations of chemical oscillators". Sci., 323(5914):614-617, 2009.

[26] A. F. Taylor, M. R. Tinsley, F. Wang, and K. Showalter. "Phase clusters in large populations of chemical oscillators". Angewandte Chemie, 123(43):10343-10346, 2011.

[27] M. R. Tinsley, S. Nkomo, and K. Showalter. "Chimera and phase-cluster states in populations of coupled chemical oscillators". Nat. Phys., 8(9):662-665, 2012.

[28] M. R. Tinsley, A. F. Taylor, Z. Huang, F. Wang, and K. Showalter. "Dynamical quorum sensing and synchronization in collections of excitable and oscillatory catalytic particles". Phys. D: Nonlinear Phenom., 239(11):785-790, 2010.

[29] M. R. Tinsley, A. F. Taylor, Z. Y. Huang, and K. Showalter. "Emergence of collective behavior in groups of excitable catalyst-loaded particles: Spatiotemporal dynamical quorum sensing". Phys. Rev. Lett., 102(15):158301, 2009. 
[30] M. Toiya, H. O. González-Ochoa, V. K. Vanag, S. Fraden, and I. R. Epstein. "Synchronization of chemical micro-oscillators". J. Phys. Chem. Lett., 1(8):1241-1246, 2010.

[31] J. F. Totz, R. Snari, D. Yengi, M. R. Tinsley, H. Engel, and K. Showalter. "Phaselag synchronization in networks of coupled chemical oscillators". Phys. Rev. E, 92(2):022819, 2015.

[32] T. J. Walker. "Acoustic synchrony: Two mechanisms in the snowy tree cricket". Sci., 166(3907):891-894, 1969.

[33] M. S. Yeung and S. H. Strogatz. "Time delay in the Kuramoto model of coupled oscillators". Phys. Rev. Lett., 82(3):648, 1999. 


\section{Chapter 2}

\section{The Belousov-Zhabotinsky Reaction}

\subsection{The Belousov-Zhabotinsky Reaction}

The Belousov-Zhabotinsky (BZ) oscillating chemical reaction was first discovered by the Russian biochemist B. P. Belousov [1] in 1950 and then further investigated by A. M. Zhabotinsky [26]. The BZ reaction has been extensively studied in the past due to its dynamical similarity to many biological systems $[13,8,12,9]$. The reaction is driven by a large negative Gibbs energy, with the overall reaction given by [21]:

$$
3 \mathrm{MA}(\mathrm{aq})+2 \mathrm{BrO}_{3}{ }^{-}(\mathrm{aq})+2 \mathrm{H}^{+}(\mathrm{aq}) \longrightarrow 2 \mathrm{BrMA}(\mathrm{aq})+4 \mathrm{H}_{2} \mathrm{O}(\mathrm{l})+3 \mathrm{CO}_{2}(\mathrm{~g}) .
$$

In general, an organic substrate, such as malonic acid (MA), is oxidized by bromate, $\mathrm{BrO}_{3}{ }^{-}$, in the presence of acid. The concentration of three intermediate species, $\mathrm{HBrO}_{2}, \mathrm{Br}^{-}$and 
the catalyst, undergo periodic changes and give rise to the observed oscillatory behavior. A detailed mechanism and mathematical model of the reaction will be described in the following section.

\subsection{The FKN Mechanism of the BZ Reaction}

In 1972, R. J. Field, E. Körös and R. M. Noyes [2] proposed the FKN mechanism for the $\mathrm{BZ}$ reaction, which consists of three main processes. In process $\mathrm{A}$, bromate is reduced to bromine, resulting in the removal of bromide. In process $\mathrm{B}$, the catalyst is oxidized via the autocatalysis of bromous acid. In process $\mathrm{C}$, the catalyst oxidizes the brominated organic species to regenerate the bromide.

Process A:

$$
5 \mathrm{Br}^{-}+\mathrm{BrO}_{3}^{-}+6 \mathrm{H}^{+} \longrightarrow 3 \mathrm{Br}_{2}+3 \mathrm{H}_{2} \mathrm{O}
$$

Process B:

$$
\mathrm{BrO}_{3}{ }^{-}+\mathrm{HBrO}_{2}+2 \mathrm{M}_{\mathrm{red}}+3 \mathrm{H}^{+} \longrightarrow 2 \mathrm{HBrO}_{2}+2 \mathrm{M}_{\mathrm{ox}}+\mathrm{H}_{2} \mathrm{O},
$$

Process C:

$$
\mathrm{MA}+2 \mathrm{M}_{\mathrm{ox}}+\mathrm{BrMA} \longrightarrow \mathrm{fBr}^{-}+2 \mathrm{M}_{\mathrm{red}}+\text { other products }
$$


where the $\mathrm{M}_{o x}$ and $\mathrm{M}_{r e d}$ represent the oxidized and reduced state of metal catalyst, respectively. $f$ is a stoichiometric factor that describes the number of bromide ions produced for every two metal catalyst ions reduced. In process $\mathrm{A}$, the inhibitor, $\mathrm{Br}^{-}$, is removed by reacting with $\mathrm{BrO}_{3}{ }^{-}$. Process $\mathrm{B}$ will initiate as the concentration of $\mathrm{Br}^{-}$falls below a critical concentration. In this process, the activator species, $\mathrm{HBrO}_{2}$, is produced autocatalytically, driving the oxidization of the metal catalyst. The rate of the autocatalysis step accelerates as the concentration of $\mathrm{HBrO}_{2}$ increases, which causes the rapid oxidization of the metal catalyst ion. Once the oxidized catalyst concentration is high enough, the inhibitor is reformed via oxidization of bromomalonic acid in process $\mathrm{C}$. This reinitiates process $\mathrm{A}$, and the cycle then repeats itself.

\subsection{ZBKE Model}

In 1993, Zhabotinsky, Buchholtz, Kiyatkin and Epstein (ZBKE) [27] developed a mathematical model of the $\mathrm{BZ}$ reaction, which included the intermediate species $\mathrm{HBrO}_{2}{ }^{+}$. The reaction scheme is written in Table 2.1. The $\mathrm{M}_{\text {red }}$ represents the reduced form of the catalyst, and $\mathrm{M}_{o x}$ represents the oxidized form of the catalyst.

In this mechanism, the reactions of RA-1 and RA-2 are rapid, so all other reactions of $\mathrm{HOBr}$ and $\mathrm{Br}_{2}$ can be neglected. Moreover, $\mathrm{CBr}_{2}(\mathrm{COOH})_{2}, \mathrm{CHOH}(\mathrm{COOH})_{2}$ and $\mathrm{CO}(\mathrm{COOH})_{2}$ are considered as final products and the equilibrium of reaction R5c is completely shifted to 
Table 2.1: ZBKE model reaction scheme

$\mathrm{H}^{+}+\mathrm{Br}^{-}+\mathrm{HOBr} \rightleftarrows \mathrm{Br}_{2}+\mathrm{H}_{2} \mathrm{O}$
$\mathrm{H}^{+}+\mathrm{Br}^{-}+\mathrm{HBrO}_{2} \rightleftarrows 2 \mathrm{HOBr}$


the right [27]. Based on these assumptions, a simplified mathematical model can be written as

$$
\frac{d X}{d t}=-k_{2} h_{0} X Y+k_{3} h_{0} X Y-2 k_{4}^{*} X^{2}-k_{5} h_{0} A X+K_{-5} U^{2}+K_{6} U(C-Z)-K_{-6} X Z
$$

$$
\begin{gathered}
\frac{d Y}{d t}=-k_{2} h_{0} X Y-k_{3} h_{0} A Y+k_{8}^{\prime} R_{1}+k_{9} B, \\
\frac{d U}{d t}=2 k_{5} h_{0} A X-2 k_{-5} U^{2}-K_{6} U(C-Z)+k_{-6} X Z, \\
\frac{d Z}{d t}=k_{6} U(C-Z)-k_{-6} X Y-k_{7} B Z+k_{-7} h_{0} R_{1}(C-Z), \\
\frac{d R_{1}}{d t}=k_{7} B Z-k_{-7} h_{0} R_{1}(C-Z)-k_{8}^{\prime} R_{1}-k_{11} R_{1} R_{2}, \\
\frac{d R_{2}}{d t}=k_{8}^{\prime} R_{1}-2 k_{10} R_{2}^{2}-k_{11} R_{1} R_{2},
\end{gathered}
$$

where the following definitions are used: $\mathrm{X}=\left[\mathrm{HBrO}_{2}\right], \mathrm{Y}=\left[\mathrm{Br}^{-}\right], \mathrm{Z}=\left[\mathrm{M}_{o x}\right], \mathrm{U}=\left[\mathrm{HBrO}_{2}^{+}\right]$, $\mathrm{R}_{1}=\left[\mathrm{CBr}(\mathrm{COOH})_{2}^{\bullet}\right], \mathrm{R}_{2}=\left[\mathrm{COH}(\mathrm{COOH})_{2}^{\bullet}\right], \mathrm{A}=\left[\mathrm{HBrO}_{3}\right]=\mathrm{h}_{0}\left[\mathrm{NaBrO}_{3}\right]_{0} /\left(0.2+\mathrm{h}_{0}\right), \mathrm{B}=$ $\left[\mathrm{CHBr}(\mathrm{COOH})_{2}\right], \mathrm{C}=\mathrm{Z}+\left[\mathrm{M}_{r e d}\right], \mathrm{h}_{0}=$ Hammet acidity function, $\mathrm{k}_{4}^{*}=\mathrm{k}_{4}\left(1+0.87 \mathrm{~h}_{0}\right)$, and $\mathrm{k}_{-5}$ $=\left(\mathrm{k}_{-5 b} \mathrm{k}_{-5 c}\right) /\left(\mathrm{k}_{5 c} \mathrm{~h}_{0}\right)$. Further simplification can be made by applying the quasi-steady-state 
approximation to R2 and assuming that radical reactions R10 and R11 are very fast. Then the rate equation of $\mathrm{R} 1$ is simplified to:

$$
\frac{d R_{1}}{d t}=k_{7} B Z-k_{-7} h_{0} R_{1}(C-Z)-\frac{k_{8}^{\prime} R_{1}}{q\left(R_{1}\right)},
$$

where

$$
\frac{1}{q\left(R_{1}\right)}=1-\frac{k_{11}^{2} R_{1}}{4 k_{8}^{\prime} k_{10}}\left(1-\left(1+\frac{8 k_{8}^{\prime} k_{10}^{\prime}}{k_{11}^{2} R_{1}}\right)^{1 / 2}\right) .
$$

The parameter $q\left(R_{1}\right)$ can be varied from 0.5 to 1.0 . If R10 reacts much faster than R11, then $q\left(R_{1}\right)=1$, and if $\mathrm{R} 10$ reacts much slower than $\mathrm{R} 11$, then $q\left(R_{1}\right)=0.5$ [27]. If the intermediate species $\mathrm{HBrO}_{2}{ }^{+}$is assumed to be at a steady state $\left(U_{s s}\right)$, then the above differential equations are simplified to the following three-variable mathematical model [22]:

$$
\begin{gathered}
\frac{d X}{d t}=-k_{2} h_{0} X Y+k_{3} h_{0} X Y-2 k_{4}^{*} X^{2}-k_{5} h_{0} A X+k_{-5} U_{s}^{2} s+k_{6} U_{s s}(C-Z)-k_{-6} X Z, \\
\frac{d Y}{d t}=-k_{2} h_{0} X Y-k_{3} h_{0} A Y+q \frac{k_{7} k_{8} B Z}{k_{-7} h_{0}(C-Z)+k_{8}}+k_{9} B \\
\frac{d Z}{d t}=k_{6} U_{s s}(C-Z)-k_{-6} X Z-\frac{k_{7} k_{8} B Z}{k_{-7} h_{0}(C-Z)+k_{8}}, \\
U_{s s}=\frac{1}{4 k_{-5}}\left(k_{6}(C-Z)+\left(k_{6}^{2}(C-Z)^{2}+16 k_{-5} k_{5} h_{0} A X+8 k_{-5} k_{-6} X Z\right)^{1 / 2}\right)
\end{gathered}
$$


This system of equations can be non-dimensionalization using the appropriate scalings shown in Table 2.2. The system of differential equations is then given by [22]:

$$
\begin{gathered}
\frac{d x}{d \tau}=\frac{1}{\epsilon_{1}}\left(-x^{2}-x+\epsilon_{2} \gamma u_{s s}^{2}+u_{s s}(1-z)-\delta x z+\mu y-x y\right), \\
\frac{d y}{d \tau}=\frac{1}{\epsilon_{4}}\left(-x y-\mu y+q \frac{\alpha z}{\epsilon_{3}+1-z}+\beta\right) \\
\frac{d z}{d \tau}=u_{s s}(1-z)-\delta x z-\frac{\alpha z}{\epsilon_{3}+1-z}
\end{gathered}
$$

where the dimensionless variables $\mathrm{x}, \mathrm{y}$ and $\mathrm{z}$ represent bromous acid, bromide and the oxidized form of the metal catalyst, respectively. $u_{s s}$ is the steady state concentration of $\mathrm{HBrO}_{2}{ }^{+}$.

\subsection{The Photosensitive BZ Reaction and Modified ZBKE Model}

In 1983, the effects of illumination on the ruthenium-complex catalyzed BZ reaction were investigated by V. Gáspár et al. [3]. They observed that the period and amplitude of the oscillation in the ruthenium-complex catalyzed system are altered by visible light. The illumination effects on the ruthenium-catalyzed BZ reaction are sensitive to the recipe $[5,25]$ 
Table 2.2: Scalings for the three variable ZBKE model

\begin{tabular}{c|c}
\hline Variable & Scaling \\
\hline $\mathrm{X}$ & $k_{5} h_{0} A x / 2 k_{4}$ \\
$\mathrm{Y}$ & $k_{5} A y / k_{2}$ \\
$\mathrm{Z}$ & $\mathrm{Cz}$ \\
$\mathrm{U}$ & $\left(k_{5} h_{0} A\right)^{2} \mu / 2 k_{4} k_{6} C$ \\
$\mathrm{t}$ & $2 k_{4} C \tau /\left(k_{5} h_{0} A x\right)^{2}$ \\
\hline Parameter & Scaling \\
\hline$\epsilon_{1}$ & $h_{0} A x / 2 k_{4} C$ \\
$\epsilon_{2}$ & $\left(k_{5} h_{0} A\right)^{2} / 2 k_{4} k_{6} C$ \\
$\epsilon_{3}$ & $k_{8} / k_{-7} h_{0} C$ \\
$\epsilon_{4}$ & $k_{5} A / k_{2} C$ \\
$\alpha$ & $2 k_{4} k_{7} k_{8} B / k_{5}^{2} k_{-7} h_{0}^{3} A^{2}$ \\
$\beta$ & $2 k_{4} k_{9} B /\left(k_{5} h_{0} A\right)^{2}$ \\
$\mu$ & $2 k_{3} k_{4} / k_{2} k_{5} h_{0}$ \\
$\gamma$ & $k_{-5} / k_{6}$ \\
$\delta$ & $k_{-6} C / k_{5} h_{0} A$ \\
&
\end{tabular}

and light intensity $[11,16,15]$. S. Kádár et al. [6] studied the reaction mechanism of the light sensitive $\mathrm{Ru}(\mathrm{bpy})_{3}^{2+} \mathrm{BZ}$ reaction and showed that the photosensitivity involves two different photochemical processes [6]: photoexcitation and photoinhibition. Illumination at wavelength $460 \mathrm{~nm}$ excites the $\mathrm{Ru}(\mathrm{bpy})_{3}^{2+}$ to produce the strong reducing agent $\mathrm{Ru}(\mathrm{bpy})_{3}^{2+*}$, which is able to reduce $\mathrm{BrO}_{3}^{-}$and $\mathrm{BrMA}$ to produce $\mathrm{HBrO}_{2}$ and $\mathrm{Br}^{-}$, respectively. The former reaction pathway, photoexcitation, is given by the following $[7,4]$ :

$$
\mathrm{Ru}(\mathrm{bpy})_{3}^{2+*}+\mathrm{Ru}(\mathrm{bpy})_{3}^{2+}+\mathrm{BrO}_{3}^{-}+3 \mathrm{H}^{+} \longrightarrow 2 \mathrm{Ru}(\mathrm{bpy})_{3}^{3+}+\mathrm{HBrO}_{2}+\mathrm{H}_{2} \mathrm{O} \text {. }
$$

The $\mathrm{BrO}_{3}^{-}$is reduced to $\mathrm{HBrO}_{2}$ via formation of $\mathrm{Ru}(\mathrm{bpy})_{3}^{3+}$. The increased concentration of 
$\mathrm{HBrO}_{2}$ initiates the autocatalytic step, which gives rise to a phase advance of the oscillators. The photoinhibition reaction is written as follows [6]:

$$
\mathrm{Ru}(\mathrm{bpy})_{3}^{2+*}+\mathrm{BrMA} \longrightarrow \mathrm{Ru}(\mathrm{bpy})_{3}^{3+}+\mathrm{Br}^{-}+\text {organic products. }
$$

In this process, the inhibitor, $\mathrm{Br}^{-}$, is produced. The addition of bromide affects process $\mathrm{A}$ in the FKN mechanism and can suppress the oscillations. This is because it takes a longer time to consume the higher bromide to the critical concentration, which leads to a longer period of the oscillation. The reaction favors either the photoinhibition or photoexcitation channel depending on the light intensity and composition of the system. The photoexcitatory system is utilized in the current work. The ZBKE model, which was described in the previous section, can be modified to account for the photosensitive reactions. V. K. Vanag et al. [24] introduced a $\phi$ term into the ZBKE model to represent the photochemical reactions. The three-variable, dimensionless photosensitive ZBKE mathematical model is given as follows:

$$
\begin{gathered}
\frac{d x}{d \tau}=\frac{1}{\epsilon_{1}}\left(\phi-x^{2}-x+\epsilon_{2} \gamma u_{s s}^{2}+u_{s s}(1-z)-\delta x z+\mu y-x y\right), \\
\frac{d y}{d \tau}=\frac{1}{\epsilon_{4}}\left(-x y-\mu y+q \frac{\alpha z}{\epsilon_{3}+1-z}+\beta\right), \\
\frac{d z}{d \tau}=\phi+u_{s s}(1-z)-\delta x z-\frac{\alpha z}{\epsilon_{3}+1-z} .
\end{gathered}
$$




\subsection{Experimental Setup}

Figure 2.1(a) shows the main components of the experimental setup, including the reactor where the BZ reaction takes place, the spatial light modulator (SLM) to control the illumination and the charge-coupled device (CCD) camera to monitor the dynamics of the reaction. A catalyst-free $\mathrm{BZ}$ solution in a volumetric flask is placed in an ice bath and is continuously pumped into the reactor at a flow rate of $1.5 \mathrm{ml} / \mathrm{min}$. The water bath maintains the temperature of the fresh solution at $21^{\circ} \mathrm{C}$ and the solution is then pumped into the reactor, which is well mixed by the stirrer. The solution is also pumped out at the same flow rate to keep a constant depth of solution. The preparation of the BZ solution is discussed in the following section.

\subsubsection{Preparation of BZ Solution and Catalyst-Loaded Beads}

The catalyst-free BZ solution is prepared by mixing the appropriate amount of malonic acid (MA), sodium bromide $(\mathrm{NaBr})$, sulfuric acid $\left(\mathrm{H}_{2} \mathrm{SO}_{4}\right)$ and sodium bromate $\left(\mathrm{NaBrO}_{3}\right)$ so that the system is a photoexcitatory BZ solution. In the present work, the catalyst-free BZ solution consists of $0.08 \mathrm{M}$ malonic acid, 0.02 M sodium bromide, $0.9 \mathrm{M}$ sulfuric acid and

$0.48 \mathrm{M}$ sodium bromate. The catalyst-loaded beads are prepared by mixing 3.0 grams of 
ion exchange resin (DOWEX 50WZ2-100) with $1.0 \mathrm{ml}$ tris(bipyridine)ruthenium(II) chloride solution $(25.0 \mathrm{mM})$. The mixture is stirred for 24 hours and is filtered using filter paper until the beads are completely dry. The dry beads are stored in a glass vial, keeping them away from light.

\subsubsection{Preparation of Large Oscillator Array}

In order to investigate collective behavior of a large number of oscillators, we designed a new reactor that can hold up to 2000 oscillators in one reaction container. This large oscillator array (LOA) is prepared by positioning the beads (diameter around $250 \mu \mathrm{m}$ ) onto an acrylic plate drilled with micrometer conical holes (slightly larger than the bead diameter). The distance between each hole is larger than two diameters of the bead to prevent local diffusive coupling. The beads are then transferred to a Teflon substrate which is covered by polydimethylsiloxane (PMDS). The PMDS is prepared by thoroughly mixing 10:1 weight ratio of dimethylsiloxane gel with the curing agent (SYLGARD184) and left at room temperature for 24 hours. The LOA is placed into the reactor vessel, which is located directly below the camera at a distance of $30 \mathrm{~cm}$. The Figure 2.1(b) shows a typical example of the LOA recorded by the CCD camera. 


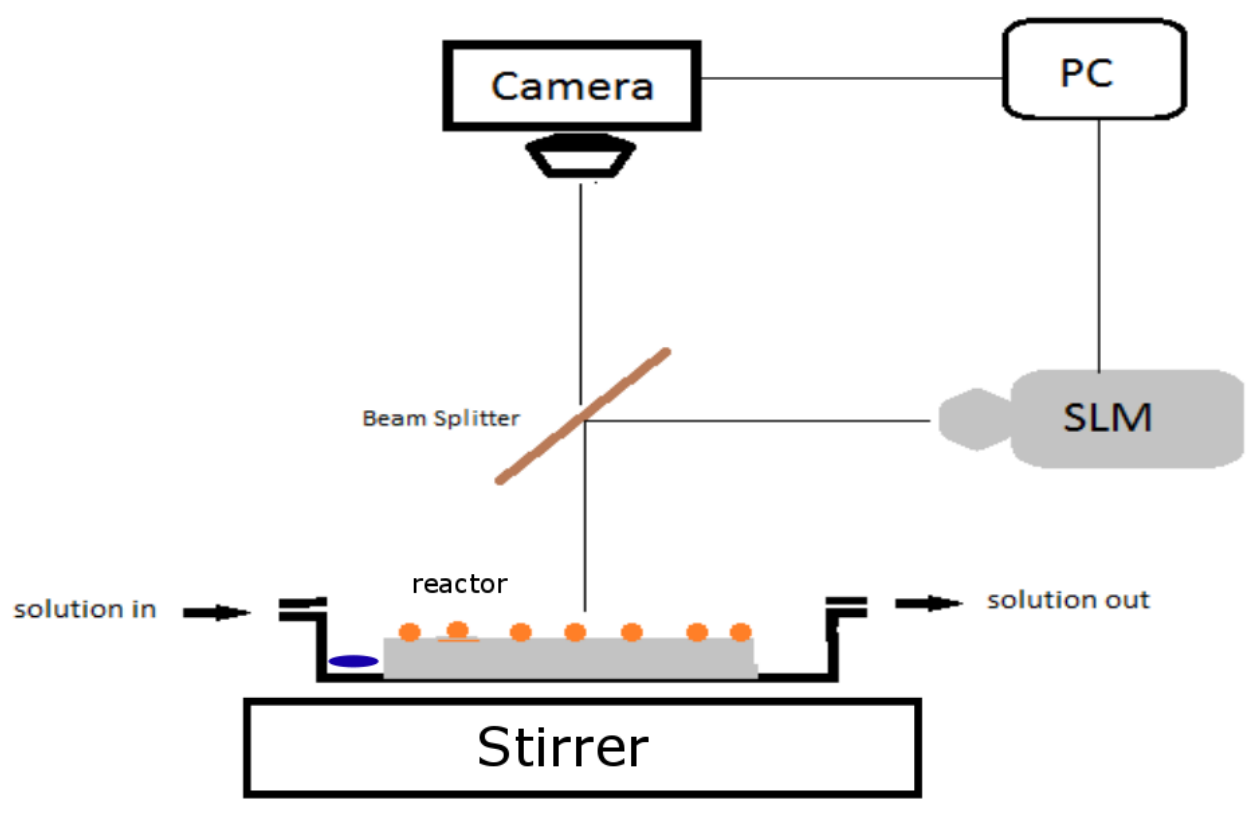

(a)

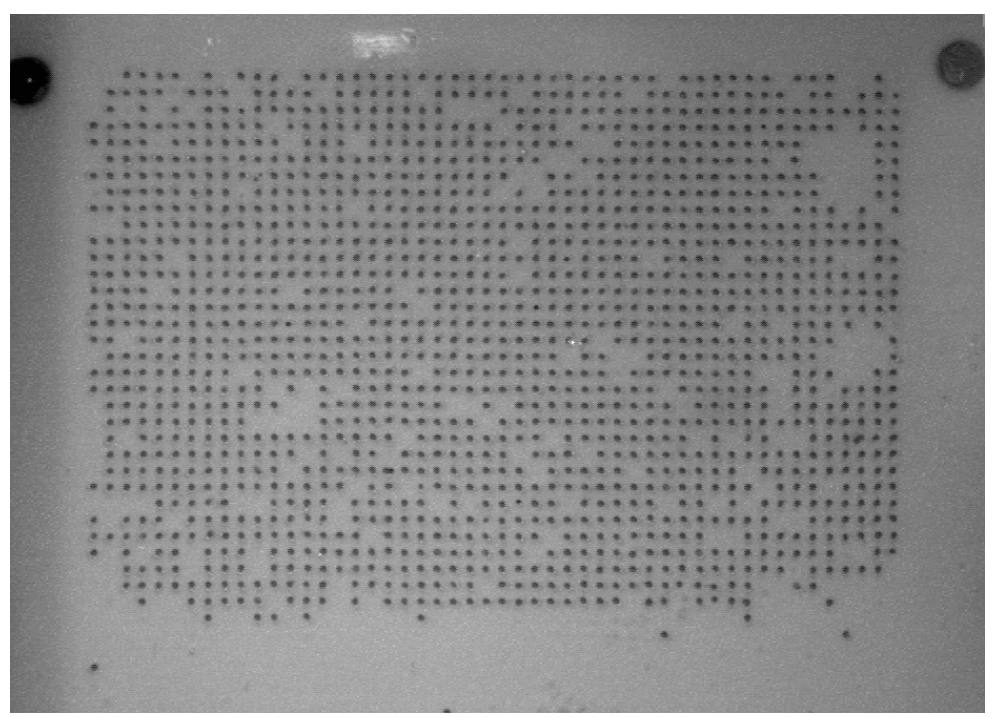

(b)

Figure 2.1: (a) Experimental setup: charge-coupled device (CCD) camera records the dynamics of each individual oscillator. The image is processed by the computer and the feedback illumination is applied to the oscillators via a spatial light modulator (SLM). (b) The large oscillator array (LOA) is recorded by the CCD camera. 


\subsubsection{Experimental Process}

The coupling method used in this experiment is light-based feedback [20, 23, 19]. The experiment is conducted using a duty cycle of $3.4 \mathrm{~s}$ and is controlled by Matlab. The reaction image is taken by a CCD camera every cycle under background light with intensity of 0.5 $\mathrm{m} \mathrm{W} \mathrm{cm}^{-2}$. The changes of transmitted light intensity of individual oscillators are recorded over time. In the catalyst-free BZ solution, the ruthenium micro-beads periodically change color between green $\left(\mathrm{Ru}(\mathrm{bpy})_{3}^{3+}\right)$ and orange $\left(\mathrm{Ru}(\mathrm{bpy})_{3}^{2+}\right)$, giving rise to different transmitted light intensities. In order to simplify the image analysis, the RGB image is converted to B \& W. The measured intensity is then used to calculate the feedback illumination by a specific algorithm depending on the experiment. The specific feedback calculations are discussed in Chapter 3. The SLM is used to illuminate each individual oscillator. The illumination intensity range is between $0.04 \mathrm{~mW} \mathrm{~cm}^{-2}$ and $2.11 \mathrm{~mW} \mathrm{~cm}^{-2}$. A typical time series of an oscillator under the background illumination is shown in Figure 2.2.

\subsection{Phase Response Curve}

The response of an oscillator to an external perturbation can be described using a phase response curve (PRC). The external perturbation can take various forms, including light, chemical and temperature stimuli. A PRC illustrates the phase change of an oscillator following a perturbation at a given phase. The PRC provides useful information to analyze the dynamics of oscillators and is widely applied in circadian rhythms $[17,10]$ and neuroscience 


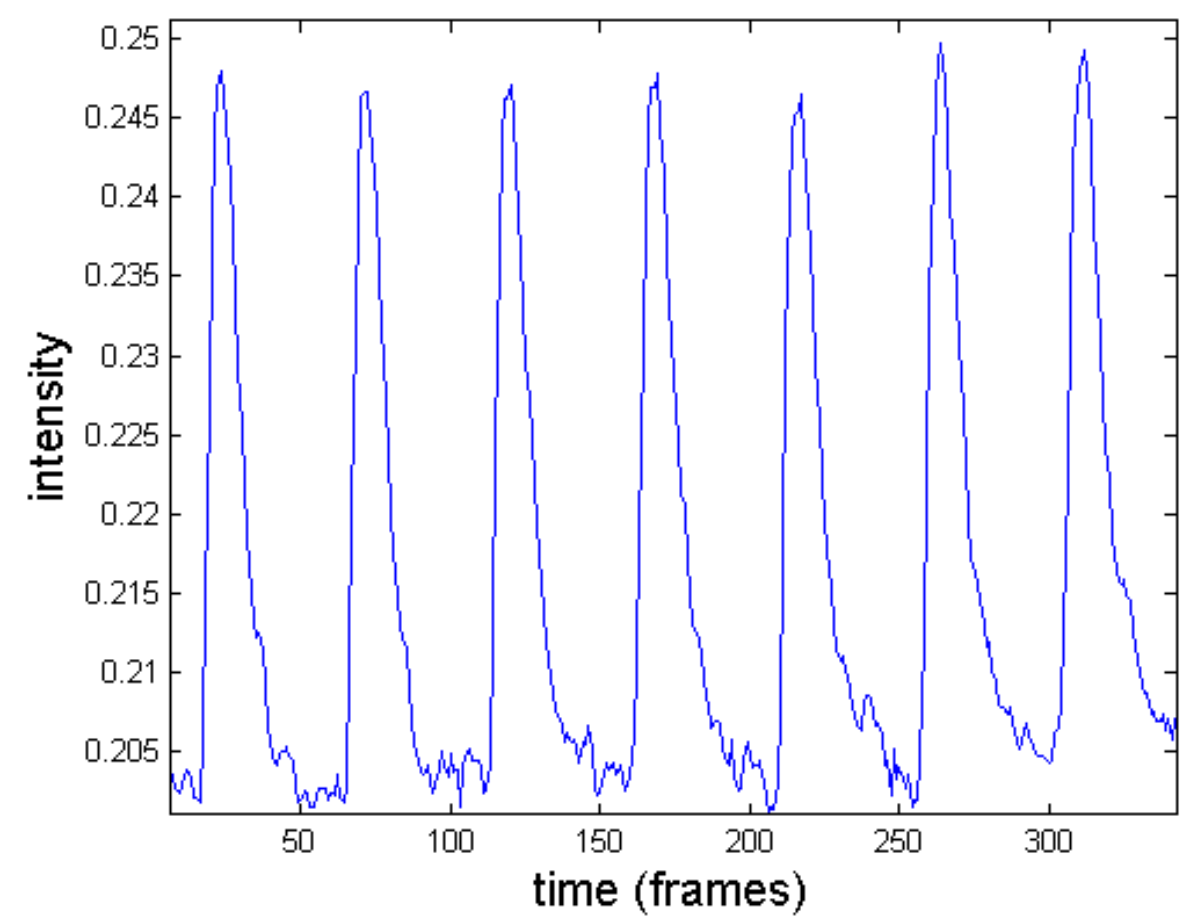

Figure 2.2: Time series showing transmitted light intensity

$[18,14]$. Our PRC experiment is conducted with the setup described in Section 2.5, and the external perturbation is high-intensity light. The period of unperturbed oscillator is $T_{0}$ and the phase, $\phi(t)$, at the perturbation is measured between 0 and $2 \pi$ using following equation:

$$
\phi(t)=2 \pi\left(T-T_{\text {peak }}\right) / T_{0}
$$

where $\phi$ is the phase of the oscillator, $T$ is the time of perturbation and $T_{\text {peak }}$ is the time of the most recent peak. The transient change of the phase of an oscillator following a perturbation is quantified by comparing the period difference between perturbed and unperturbed cycles. 
The magnitude of the phase changes $\Delta \phi$ is given by the equation:

$$
\Delta \phi=2 \pi\left(T_{0}-T_{1}\right) / T_{0},
$$

where the $T_{1}$ is the period of the cycle with the perturbation. If $\Delta \phi<0$, the cycle becomes longer after a perturbation and is called a phase retardation. If $\Delta \phi>0$, the oscillator has a phase advance. The phase has no shift when $\Delta \phi=0$.

\subsubsection{PRC Experimental Procedure and Results}

The PRC experiment is performed using the large oscillator array BZ reaction. The background light is initially applied and the beads oscillate at their natural periods. At an arbitrary time, high-intensity light is switched on for $3.0 \mathrm{~s}$ and then switched back to the background light intensity. The large number of oscillators have random initial phases, and the phases prior to the perturbation of the oscillators are randomly distributed between 0 to $2 \pi$. Therefore, a single perturbation is sufficient to construct the PRC. Experimental PRCs based on small and large light perturbations are shown in Figure 2.3(a). The highintensity perturbation gives rise to a larger phase advanced region, also called the phase resetting region. This is the region of the phase where oscillators are phase reset to $2 \pi$. The ZBKE photosensitive modeling results show similar featured PRCs, Figure 2.3(b). In the simulation, a small region of phase retardation (negative response) is observed. This phase 
retardation is not apparent in the experimental PRCs due to experimetnal noise.

The mechanism of the light perturbation on the photosensitive BZ reaction is described in Section 2.4. The $\mathrm{HBrO}_{2}$ and $\mathrm{Br}^{-}$have a pivotal influence on the phase advance and delay. The oscillators undergo phase advance or delay depending on the stimulus time with respect to previous peaks. In the phase advancing region, the dominant process is the $\mathrm{HBrO}_{2}$ generated along with $\mathrm{Ru}(\mathrm{bpy})_{3}^{3+}$ by the light perturbation. This gives rise to a positive phase response. Phase delay, the negative response, is due to the production of the inhibitor $\mathrm{Br}^{-}$. The photochemically generated $\mathrm{Br}^{-}$takes a longer time to be consumed than in the unperturbed system, leading to a lengthening of the period of the oscillation.

In conclusion, in the photoexcitatory system, the response of oscillators to light perturbations depends on the phase of the oscillators and the magnitude of the perturbation. The experimental and modeling PRCs characterize the oscillators' response to a pulse perturbation, in which the phase resetting region is observed. The photoexcitatory BZ system is used to investigate the echo phenomena, as discussed in the next chapter. 


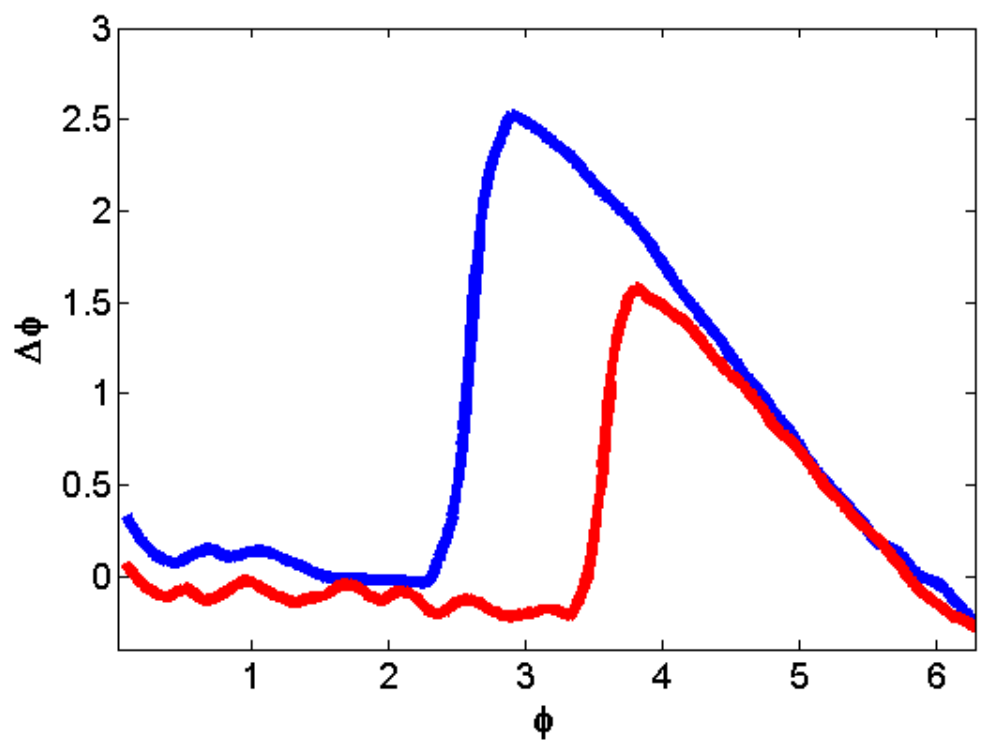

(a)

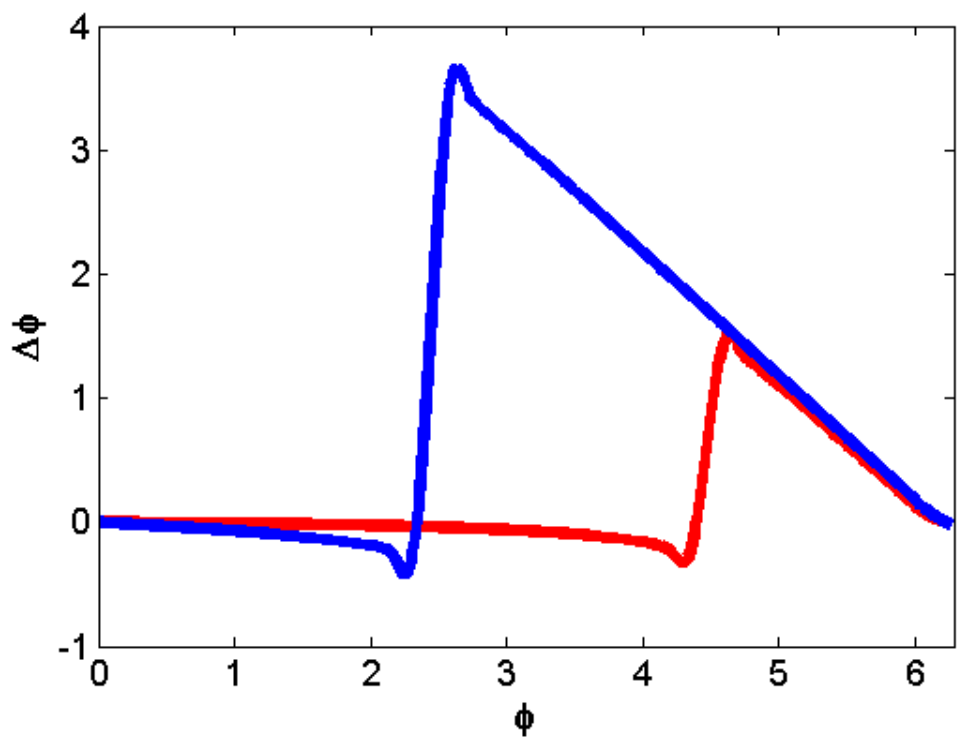

(b)

Figure 2.3: Experiment and simulation of PRCs under different sizes of simulus. (a) Experiments: (i) perturbation of high light intensity, $\phi=2.10 \mathrm{~mW} \mathrm{~cm}^{-2}$, blue; (ii) perturbation of low light intensity, $\phi=1.05 \mathrm{~mW} \mathrm{~cm}{ }^{-2}$, red. (b) Simulations: (i) high intensity perturbation, $\phi=0.147$, blue; (ii) low intensity perturbation, $\phi=0.0218$, red. 


\section{Bibliography}

[1] B. P. Belousov. "A periodic reaction and its mechanism". Compil. Abstr. on Radiat. Medicine, $\mathbf{1 4 7}(1): 145,1959$.

[2] R. J. Field, E. Körös, and R. M. Noyes. "Oscillations in chemical systems. II. Thorough analysis of temporal oscillation in the bromate-cerium-malonic acid system". J. Am. Chem. Soc., 94(25):8649-8664, 1972.

[3] V. Gáspár, G. Bazsa, and M. Beck. "The influence of visible light on the Belousov-Zhabotinskii oscillating reactions applying different catalysts". Zeitschrift Fur Physikalische Chemie-Leipzig, 264(1):43-48, 1983.

[4] I. Hanazaki, A. Kaminaga, and Y. Mori. "Response of some chemical oscillators to the pulsed light perturbation". ACH, models chemistry, 135(3):257-268, 1998.

[5] I. Hanazaki, Y. Mori, T. Sekiguchi, and G. Rábai. "Photo-response of chemical oscillators". Phys. D: Nonlinear Phenom., 84(1):228-237, 1995. 
[6] S. Kádár, T. Amemiya, and K. Showalter. "Reaction mechanism for light sensitivity of the $\mathrm{Ru}(\mathrm{bpy})_{3}^{2+}$-catalyzed Belousov-Zhabotinsky reaction". J. Phys. Chem. A, 5639(97):8200-8206, 1997.

[7] A. Kaminaga and I. Hanazaki. "Transient photoresponse of the tris $(2,2$ 'bipyridine)ruthenium(II)-catalyzed minimal bromate oscillator". J. Phys. Chem. A, 102(19):3307-3314, 1998.

[8] K. J. Lee, E. C. Cox, and R. E. Goldstein. "Competing patterns of signaling activity in Dictyostelium discoideum". Phys. Rev. Lett., 76(7):10, 1996.

[9] M. Markus, G. Kloss, I. Kusch, et al. "Disordered waves in a homogeneous, motionless excitable medium". Nat., 371(6496):402-404, 1994.

[10] D. S. Minors, J. M. Waterhouse, and A. Wirzjustice. "A human phase response curve to light”. Neurosci. Lett., 133(1):36-40, 1991.

[11] Y. Mori, Y. Nakamichi, T. Sekiguchi, N. Okazaki, T. Matsumura, and I. Hanazaki. "Photo-induction of chemical oscillation in the Belousov-Zhabotinsky reaction under the flow condition". Chem. Phys. Lett., 211(4-5):421-424, 1993.

[12] S. C. Müller, T. Mair, and O. Steinbock. "Traveling waves in yeast extract and in cultures of Dictyostelium discoideum". Biophys. Chem., 72(1):37-47, 1998.

[13] S. Nagano. "Modeling the model organism Dictyostelium discoideum". Dev. Growth \& Differ., 42(6):541-550, 2000. 
[14] H. M. Pinsker. "Aplysia bursting neurons as endogenous oscillators. I. Phase-response curves for pulsed inhibitory synaptic input". J. Neurophysiol., 40(3):527-543, 1977.

[15] M. Ram Reddy, Z. Szlavik, Z. Nagy-Ungvarai, and S. C. Müller. "Influence of light on the inorganic part of the ruthenium-catalyzed Belousov-Zhabotinsky reaction". J. Phys. Chem., 99(41):15081-15085, 1995.

[16] T. Sekiguchi, Y. Mori, and I. Hanazaki. "Photo-response of the $\left[\mathrm{Ru}(\mathrm{bpy})_{3}\right]^{2+} / \mathrm{BrO}_{3}^{-} /$ $\mathrm{H}^{+}$system in a continuous-flow stirred tank reactor". Chem. Lett., 22(8):1309-1312, 1993.

[17] M. A. St Hilaire, J. J. Gooley, S. B. S. Khalsa, R. E. Kronauer, C. A. Czeisler, and S. W. Lockley. "Human phase response curve to a $1 \mathrm{~h}$ pulse of bright white light". The J. Physiol., 590(13):3035-3045, 2012.

[18] T. Tateno and H. Robinson. "Phase resetting curves and oscillatory stability in interneurons of rat somatosensory cortex". Biophys. J., 92(2):683-695, 2007.

[19] A. F. Taylor, M. R. Tinsley, and K. Showalter. "Insights into collective cell behaviour from populations of coupled chemical oscillators". Phys. Chem. Chem. Phys., 17(31):20047-20055, 2015.

[20] M. R. Tinsley, S. Nkomo, and K. Showalter. "Chimera and phase-cluster states in populations of coupled chemical oscillators". Nat. Phys., 8(9):662-665, 2012. 
[21] R. Toth and A. F. Taylor. "The tris(2,2-bipyridyl)ruthenium-catalysed BelousovZhabotinsky reaction". Prog. React. Kinetics Mech., 31(2):59-115, 2006.

[22] R. Toth, A. F. Taylor, and M. R. Tinsley. "Collective behavior of a population of chemically coupled oscillators". J. Phys. Chem. B, 110(20):10170-10176, 2006.

[23] J. F. Totz, R. Snari, D. Yengi, M. R. Tinsley, H. Engel, and K. Showalter. "Phaselag synchronization in networks of coupled chemical oscillators". Phys. Rev. E, 92(2):022819, 2015.

[24] V. K. Vanag, L. Yang, M. Dolnik, A. M. Zhabotinsky, and I. R. Epstein. "Oscillatory cluster patterns in a homogeneous chemical system with global feedback". Nat., 406(6794):389-391, 2000.

[25] T. Yamaguchi, Y. Shimamoto, T. Amemiya, M. Yoshimoto, T. Ohmori, M. Nakaiwa, T. Akiya, M. Sato, and T. Matsumura-Inoue. "Bromomalonic acid as a source of photochemically produced $\mathrm{Br}^{-}$ion in the $\mathrm{Ru}(\mathrm{bpy})_{3}^{2+}$-catalyzed Belousov-Zhabotinsky reaction". Chem. Phys. Lett., 259(1):219-224, 1996.

[26] A. M. Zhabotinsky. "Periodic processes of malonic acid oxidation in a liquid phase". Biophys., 9(3):329-335, 1964.

[27] A. M. Zhabotinsky, F. Buchholtz, A. B. Kiyatkin, and I. R. Epstein. "Oscillations and waves in metal-ion-catalysed bromate oscillating reactions in highly oxidized states". $J$. Phys. Chem., 97(29):7578-7584, 1993. 


\section{Chapter 3}

\section{Echo Phenomena in Populations of}

\section{Chemical Oscillators}

\subsection{Introduction}

The emergence of collective dynamical behaviors in biological systems has attracted considerable attention $[15,2,4,5,3]$. The $\mathrm{BZ}$ chemical reaction has been used as an idealized model to study a number of such biological behaviors $[8,19,9,21,1]$. In the past decade, studies of populations of coupled BZ chemical oscillators have showed a variety of complex collective behaviors, such as synchronization $[8,20]$, phase clusters $[17,16]$ and the chimera state $[18,13]$. A recent report demonstrated that large populations of Kuramoto oscillators can exhibit echo behavior [14]. Echo behavior has previously been reported in a number of fields in physics, including nuclear magnetic resonance studies, cyclotron and plasma waves 
$[7,6,11]$. The recent discovery by E. Ott et al. [14] is of particular importance since it is the first demonstration of echo behavior in a biologically relevant system. In their report, a set of coupled phase oscillators was perturbed twice, at $t=0$, and at an arbitrary time $t=\tau$ later, with the oscillators rapidly phase dispersing following each perturbation. Then, at $t=2 \tau$, a measurable response is seen in the collective signal of the oscillators. The macroscopic response is quantified using a global order parameter [10]. In this work, we explore the echo phenomenon using a community of photosensitive heterogeneous BZ oscillators. This chapter includes details of the experiment and simulation results on echo phenomena. These are the first experimental studies of echo phenomena using a chemical oscillator system.

\subsection{Experiment}

\subsubsection{Experimental Measurements}

The large oscillator array (LOA) system is used to investigate the phenomenon, which permits coupling of over 1000 oscillators. The experimental setup and duty cycle are described in Section 2.5 of Chapter 2. Two coupling conditions have been investigated with this system: uncoupled and globally coupled. In the latter, the oscillators are photochemically coupled by light perturbations governed by the feedback equation as follows:

$$
\phi_{i}=\phi_{b}+\frac{k}{N} \sum_{j=1}^{N}\left(z_{j}-z_{i}\right)
$$


where $\phi_{i}$ is the feedback light intensity to the $i^{\text {th }}$ oscillator within the range of $0-255$ in grayscale units. $\phi_{b}$ is the background intensity, which is selected to be 140 in grayscale. $k$ is

the coupling strength, $N$ is the number of oscillators and the gray level is a measurement of the transmitted light intensity and is proportional to the catalyst $\mathrm{Ru}(\mathrm{bpy})_{3}^{3+}$ concentration. The order parameter $R$ is used to measure the phase coherence of the population of oscillators ( Section 1.2 of Chapter 1 ). The order parameter varies as $0 \leq R \leq 1$, where $R=1$ indicates that the oscillators are completely synchronized and $R=0$ indicates that the oscillators are completely asynchronous. When the oscillators are partial synchronized, $R$ has a value in the middle range.

\subsubsection{Experimental Results}

The experiments are carried out using the excitatory photosensitive BZ reaction oscillator in an LOA subject to two high-intensity light pulses separated by a time interval $\tau$. The global Kuramoto order parameter as a function of time from a typical experiment without global coupling is shown in Figure 3.1(a). After each light perturbation, the order parameter value suddenly increases and then exponentially decays with increasing time. The increase in the order parameter is due to the phase alignment of a portion of oscillators. These oscillators simultaneously fire since they are in the phase resetting region of their phase response curve (PRC). The value of $R$ decays with time due to the oscillator frequency heterogeneity. The echo response forms spontaneously at time $\tau$ following the second perturbation. Echo 


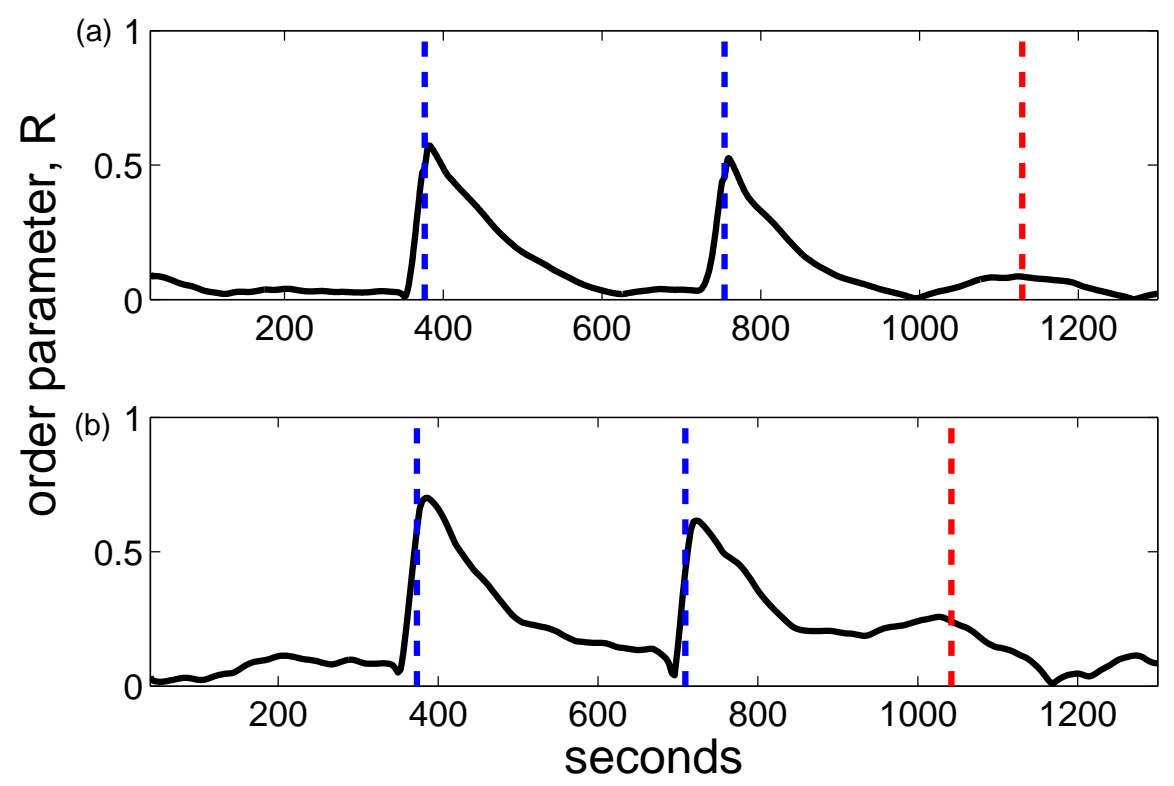

Figure 3.1: Experimental measurements illustrating echo behavior in populations of Belousov-Zhabotinsky (BZ) oscillators. The order parameter $R$ is calculated from the phases of the oscillators and plotted as a function of time. The phases of the oscillators are determined using linear interpolation between consecutive peak times. An echo is exhibited in the magnitude of the order parameter $R$ at time $\mathrm{t}_{p}+2 \tau$ for a system perturbed at times $t_{p}$ and $t_{p}+\tau$. (a) Uncoupled system, $k=0$, with $t_{p}=376 \mathrm{~s}, \tau=378 \mathrm{~s}$ and $N=1295$. (b) Coupled system, $k=0.25$, with $t_{p}=373 \mathrm{~s}, \tau=336 \mathrm{~s}$ and $N=1001$. Average natural period and standard deviation in (a) $T_{0}=36.5 \pm 3.2 \mathrm{~s}$, (b) $T_{0}=42.6 \pm 8.2 \mathrm{~s}$.

behavior in an experiment with weak coupling is shown in Figure 3.1(b). The phase dispersion of the coupled oscillators is now slower following each perturbation. An echo is again observed at time $\tau$ following the second perturbation.

To gain insight into this spontaneous response, the phase of each oscillator as a function of its frequency has been plotted in a time sequence in Figure 3.2. Figure 3.2(a) shows the 
time just before the first stimulus, in which the oscillators are randomly distributed in phase. Just after the first stimulus, the phase of the oscillators within the phase resetting region have phase advanced to accumulate near zero, as shown in Figure 3.2(b). At time $\tau / 2$, the phases of oscillators form three bands, as shown in Figure 3.2(c). With increasing time, the number of bands and their steepness increase, while their thickness decreases, as shown in Figure 3.2(d). Figure 3.2(e) shows a portion of the oscillators are phase reset at the second perturbation. The phase distribution at $2 \tau$, the time of echo, is plotted in Figure $3.2(\mathrm{f})$. The banding structure is clearly visible at time of echo. To further investigate these patterns, the ZBKE simulation is performed.

\subsection{Simulation}

\subsubsection{ZBKE model}

A nondimensional, three-variable photosensitive ZBKE model is used to simulate the experiment [22]. The dimensionless equations are modified to $\frac{d x_{i}}{d \tau}=\phi_{i}+\Phi+f_{x}\left(x_{i}, y_{i}, z_{i}\right)$, $\frac{d y_{i}}{d \tau}=f_{y}\left(x_{i}, y_{i}, z_{i}, q_{i}\right)$ and $\frac{d z_{i}}{d \tau}=\phi_{i}+\Phi+f_{z}\left(x_{i}, z_{i}\right)$. The terms $x_{i}, y_{i}, z_{i}$ and $q_{i}$ are $\mathrm{HBrO}_{2}$, $\mathrm{Br}^{-}, \mathrm{Ru}(\mathrm{bpy})_{3}^{3+}$ and stoichiometric coefficient of the $i^{\text {th }}$ oscillators respectively. $f_{x}, f_{y}$ and $f_{z}$ are rate equations of non-photosensitive ZBKE model. The term $\Phi$ is incorporated into the variables $x$ and $z$ to describe the light perturbation, as discussed in Section 2.4 of Chapter 

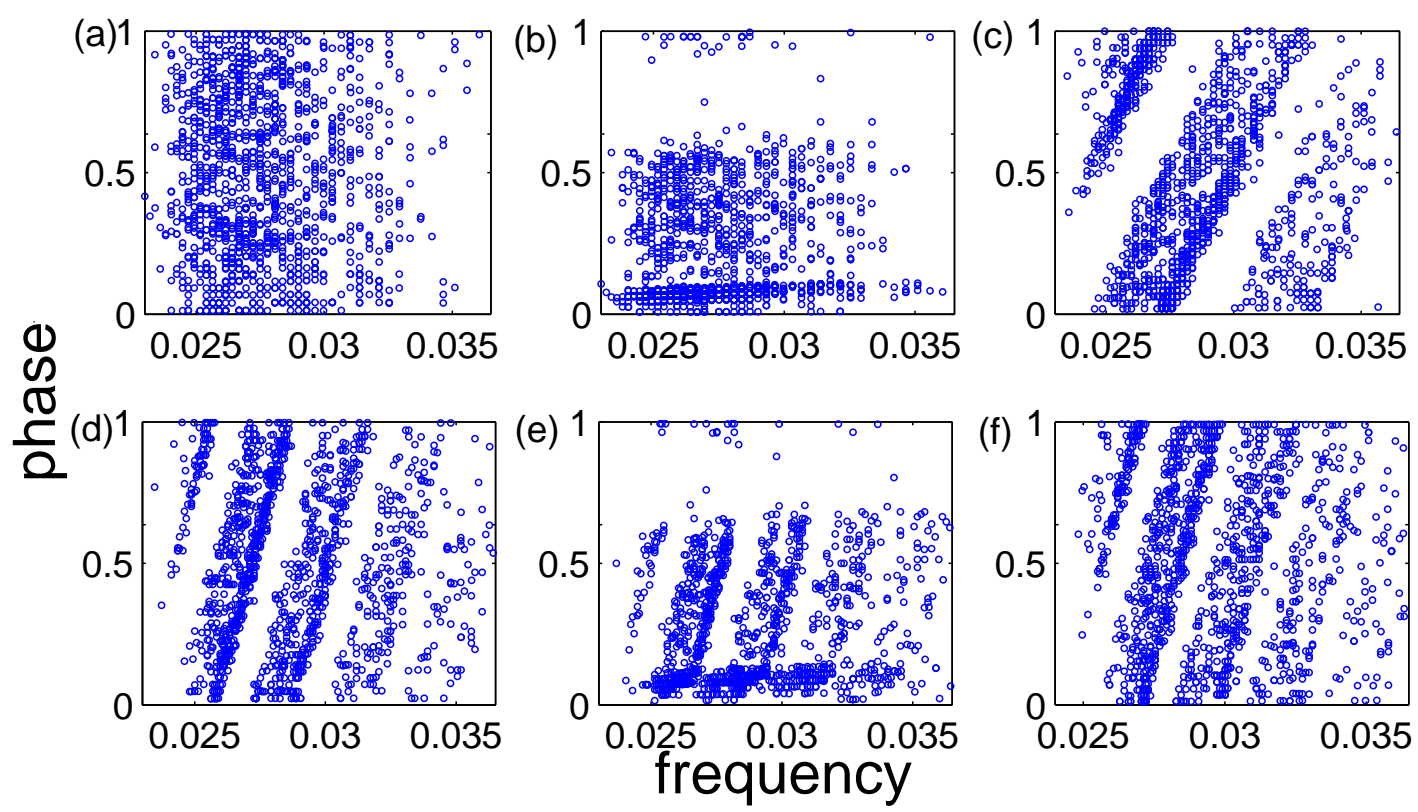

Figure 3.2: Plots of phase as a function of frequency of oscillators from the experiment shown in Figure 3.1(a) at the following times: (a) Immediately prior to first perturbation at $t_{p}$, (b) immediately following the first perturbation, in which the oscillators in the resetting region of their phase are phase reset to 0, (c) half-way between the two perturbations, $t_{p}+\tau / 2$, (d) immediately prior to the second perturbation, (e) immediately following the second perturbation, in which oscillators in the resetting region of their phase are phase reset to 0 and (f) at the time of the echo. 
2. The global coupling term, $\phi_{i}$, is realized by the equation:

$$
\phi_{i}=\frac{k}{N} \sum_{j=1}^{N}\left(z_{j}-z_{i}\right)
$$

where $k$ is the strength of coupling, $N$ is number of oscillators and variable $z_{i}$ and $z_{j}$ represents the term of $\mathrm{Ru}(\mathrm{bpy})_{3}^{3+}$ of the $i^{\text {th }}$ and the $j^{\text {th }}$ oscillators. The simulations are carried out with and without global coupling for comparison with experiment. The three coupled differential equations are numerically integrated using Euler's method, in which the step size is 0.0005 . Hetergenity is incorporated into the model using the stoichiometric coefficient $q_{i}$. The initial phase of each oscillator is set to be randomly distributed, and their natural periods are normally distributed. Simulations are carried out using up to 100,000 oscillators, with a typical period distribution of $42.5 \pm 2.4$.

\subsubsection{Simulation Results}

Figure 3.3(a) shows the order parameter $R$ as a function of time from simulations of an uncoupled system, with $k=0$. The system is perturbed by increasing the light intensity at $t_{p}=500$ and then at time $\tau=650$ later. Each perturbation is followed by rapid phase dispersion, with a corresponding decrease in the global order parameter. An increase in the order parameter is exhibited at time $t_{p}+2 \tau$, the echo, and a smaller second echo can be seen at time $t_{p}+3 \tau$ (not shown in Figure 3.3(a)). The second echo, which was predicted theoretically [14], is not typically visible in the experimental system owing to experimental noise, although we have observed it on occasion. The value of $\tau$ used in the simulation is 


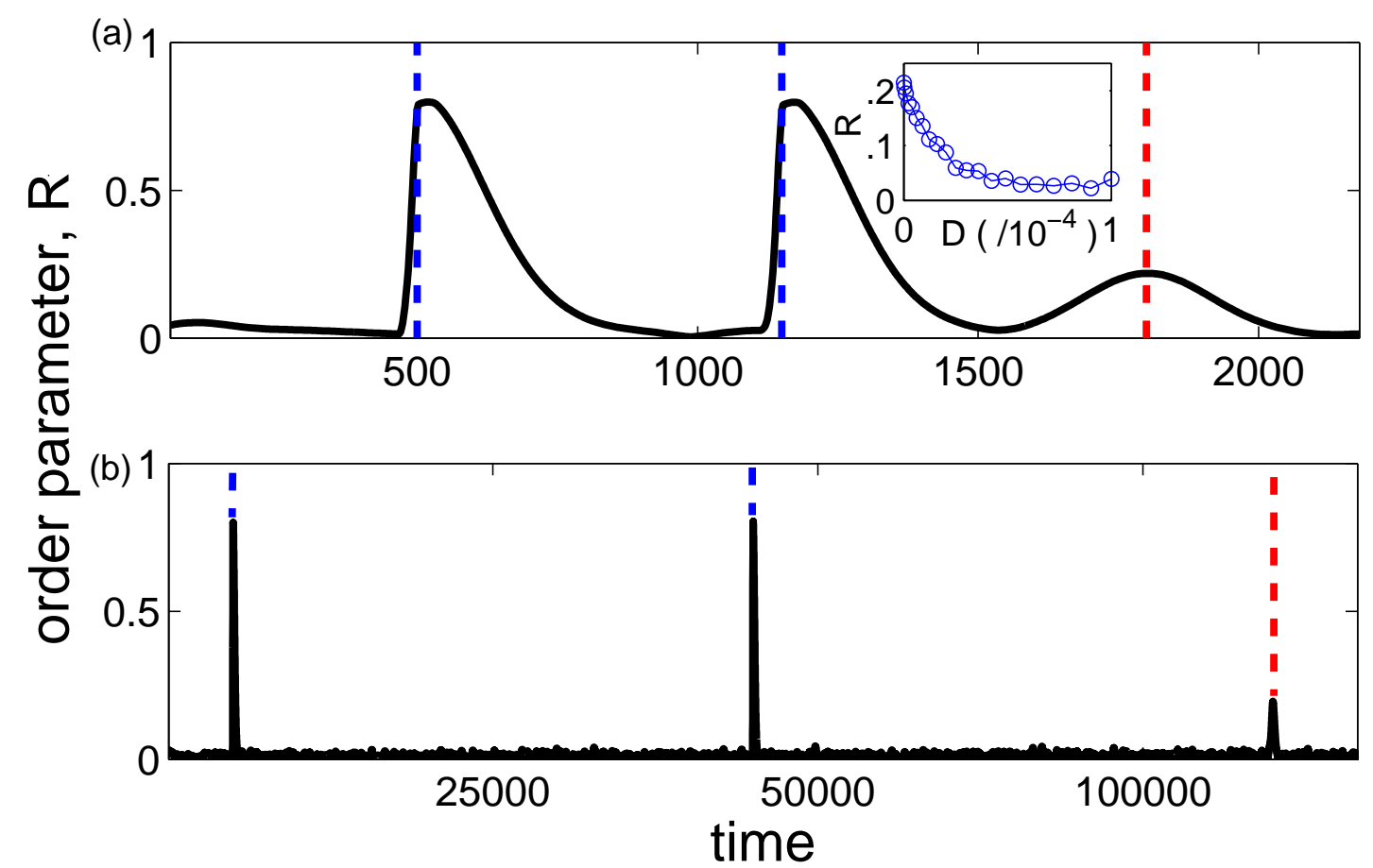

Figure 3.3: Simulations of the modified ZBKE model illustrating echo behavior in populations of uncoupled photosensitive BZ oscillators. The order parameter $R$ is calculated from the phases of the oscillators and plotted as a function of time. An echo is exhibited in the magnitude of $R$ at time $t_{p}+2 \tau$ for a system perturbed at times $t_{p}$ and $t_{p}+\tau$. (a) System with $k=0, \mathrm{t}_{p}=500, \tau=800$, and $N=5000$. Inset, magnitude of the echo as a function of the noise intensity D. (b) System with $k=0, t_{p}=5000, \tau=40000$, and $N=5000$. Average natural period $T_{0}=42.5 \pm 2.4$ (dimensionless time units).

approximately 15 times the mean period of the oscillators. Figure 3.3(b) shows that the echo phenomenon also occurs in a simulation with a much larger value of $\tau$, equal to approximately 1000 periods.

Figure 3.4(a) reveals that, as in the experimental system, the introduction of coupling 

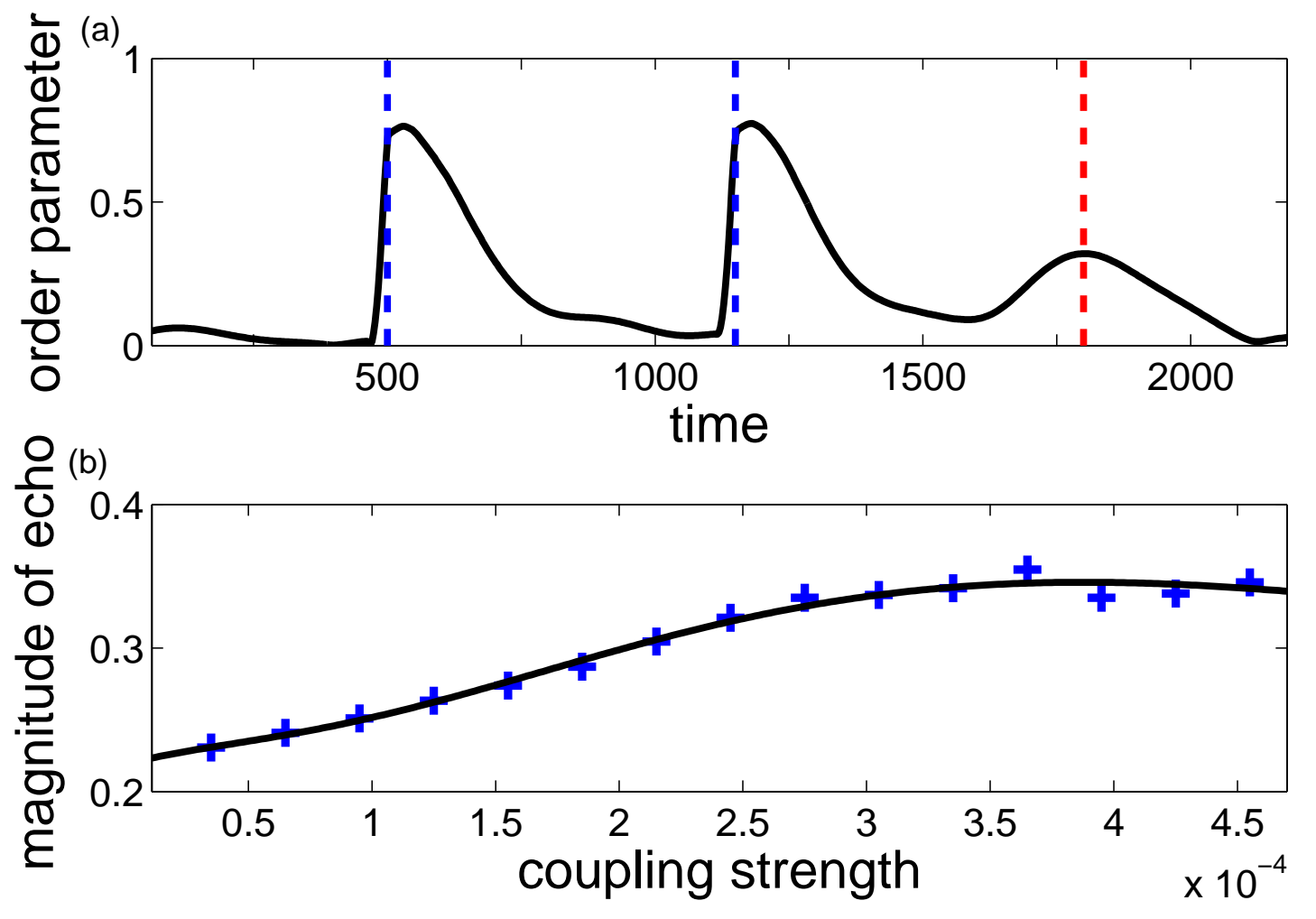

Figure 3.4: (a) Numerical simulation of a coupled system with $k=3.35 \times 10^{-4}, \tau=650$, and $N=5000$. Note the slower phase dispersion following each perturbation and the slightly larger magnitude of the echo. (b) Simulated maximum value of the order parameter at the time of the echo as a function of coupling strength.

leads to slower phase dispersion of the oscillators following each perturbation. The impact of coupling strength on the maximum magnitude of the order parameter in the vicinity of the echo is shown in Figure 3.4(b). The magnitude of the echo is an increasing function of $k$. However, at larger values of $k$, the phase dispersion is sufficiently slow that the oscillators have not fully dispersed at the time of the echo, which interferes with the observation of the echo phenomenon. 
Any experimental system has inherent noise, including our system of photosensitive BZ oscillators. The impact of such noise on the echo behavior can be explored in the numerical system by introducing additive independent Gaussian white noise to the simulation, with noise intensity D [12]. The inset in Figure 3.3(a) shows the impact increasing noise intensity, with the size of the each peak reducing with increasing noise intensity. At a larger magnitude of noise, the echo phenomenon is no longer observed.

Another factor impacting the magnitude of the echo is the size of the perturbation. The dependence on the magnitude of the perturbation is shown in Figure 3.5(a). The xaxis of this figure is defined by measuring the size of the associated phase resetting region of the phase response curve (PRC) of the oscillators for a given size perturbation. The PRC has been discussed in Section 2.6 of Chapter 2. The y-axis is determined using a perturbation of the corresponding magnitude in the echo simulation and measuring the maximum value of the order parameter in the vicinity of the echo. Figure 3.5(b) shows two PRCs constructed from simulations using large and small stimuli, with the size of the phase resetting region, $X$, indicated in both graphs. Since the size of the phase resetting region is a monotonically increasing function of the stimulus size, as shown in Figure 3.5(a), we are effectively illustrating the size of the echo as a function of the perturbation size. This plot shows the maximum echo response for a value of $X=0.6$.

As a comparison to the experimental results, the phase of each oscillator as a function of its frequency at various times during simulation is plotted in Figure 3.6. Prior to the first perturbation, the oscillators (plotted as blue dots) are distributed randomly in phase, Figure 

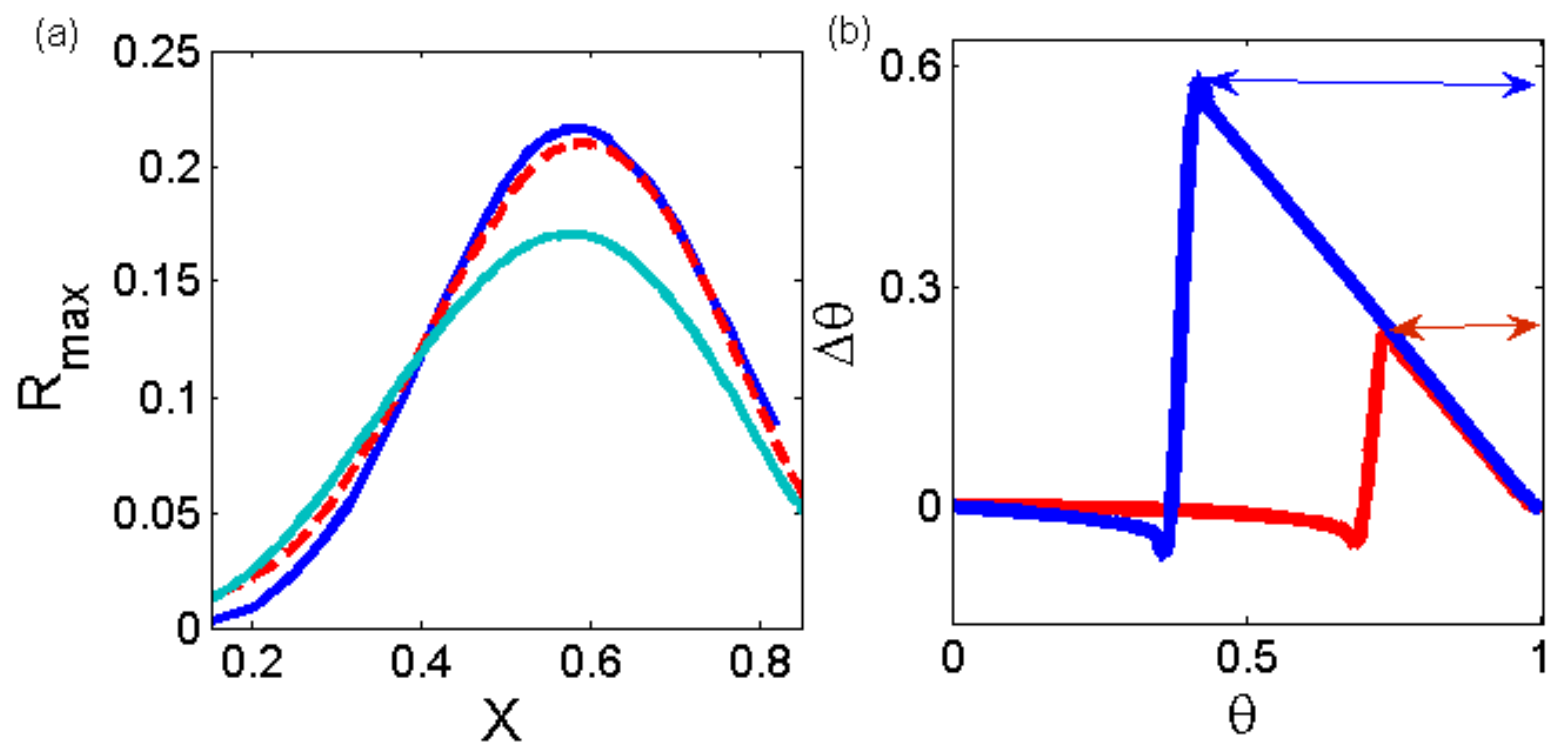

Figure 3.5: Magnitude of the simulated order parameter $R$ at the time of the echo as a function of the size of the phase resetting region, $X$, which increases monotonically with the size of the perturbation. ZBKE simulation values (dark blue line), values calculated using groups I and II (light blue line), values calculated using groups I - IV (red dashed line). (b) Simulated phase response curves for stimulus $\Phi_{\text {perturb }}=0.147$ (blue line) and $\Phi_{\text {perturb }}=$ 0.0218 (red line). The size of the associated phase resetting regions is shown by the red and blue horizontal arrows, respectively. Simulation parameters as in Figure 3.3.

3.6(a). Immediately following the first perturbation, the oscillators that were in the resetting region of their phase are phase reset to zero, Figure 3.6(b), where the reset oscillators are plotted as red dots overlying the unreset oscillators plotted as blue dots. The oscillators then advance in phase until all of the phases of the oscillators that were perturbed, as well as the phases of the oscillators that were not perturbed, are distributed along a series of bands. The steepness and number of the bands increase with time, while their thickness decreases. 
This can be seen in Figure 3.6(c) (which occurs halfway between the two perturbations) compared to Figure 3.6(d) (which occurs immediately prior to the second perturbation). At the time of the second perturbation, $t_{p}+\tau$, any oscillator lying in the resetting region is phase reset to zero, as shown Figure 3.6(e), where the red (blue) oscillators of Figure 3.6(d) that are reset are plotted in Figure 3.6(e) with their color changed from red (blue) to green (black). At the time of the echo, $t_{p}+2 \tau$, the oscillators are distributed along either a thin set of bands or a set of short steeper band segments that appear to branch off of the primary bands, Figure 3.6(f). Note that the four colors appearing in Figure 3.6(e),(f) correspond to four distinct groups of oscillators: group I (green) contains those oscillators that are reset by both perturbations; group II (red) contains those oscillators that are reset by the first but not the second perturbation; group III (black) contains those oscillators that are reset by the second but not the first perturbation; and group IV (blue) contains those oscillators that are not phase reset by either perturbation.

Examination of the Figure 3.6(f), there are clearly detailed structures, with the oscillators distributed along either a thin set of bands or a set of short steeper band segments that appear to branch off of the primary bands. Figure 3.6(f) reveals that there are structure and information within the phase distribution of the oscillators at the time of the echo; however, how this arises and why it leads to an increase in the order parameter at time $t_{p}+2 \tau$ is not immediately apparent.

To further our understanding, we focus on the numerical simulation and the division of the oscillator populations into four groups, as previously discussed. By tracking the behaviors 

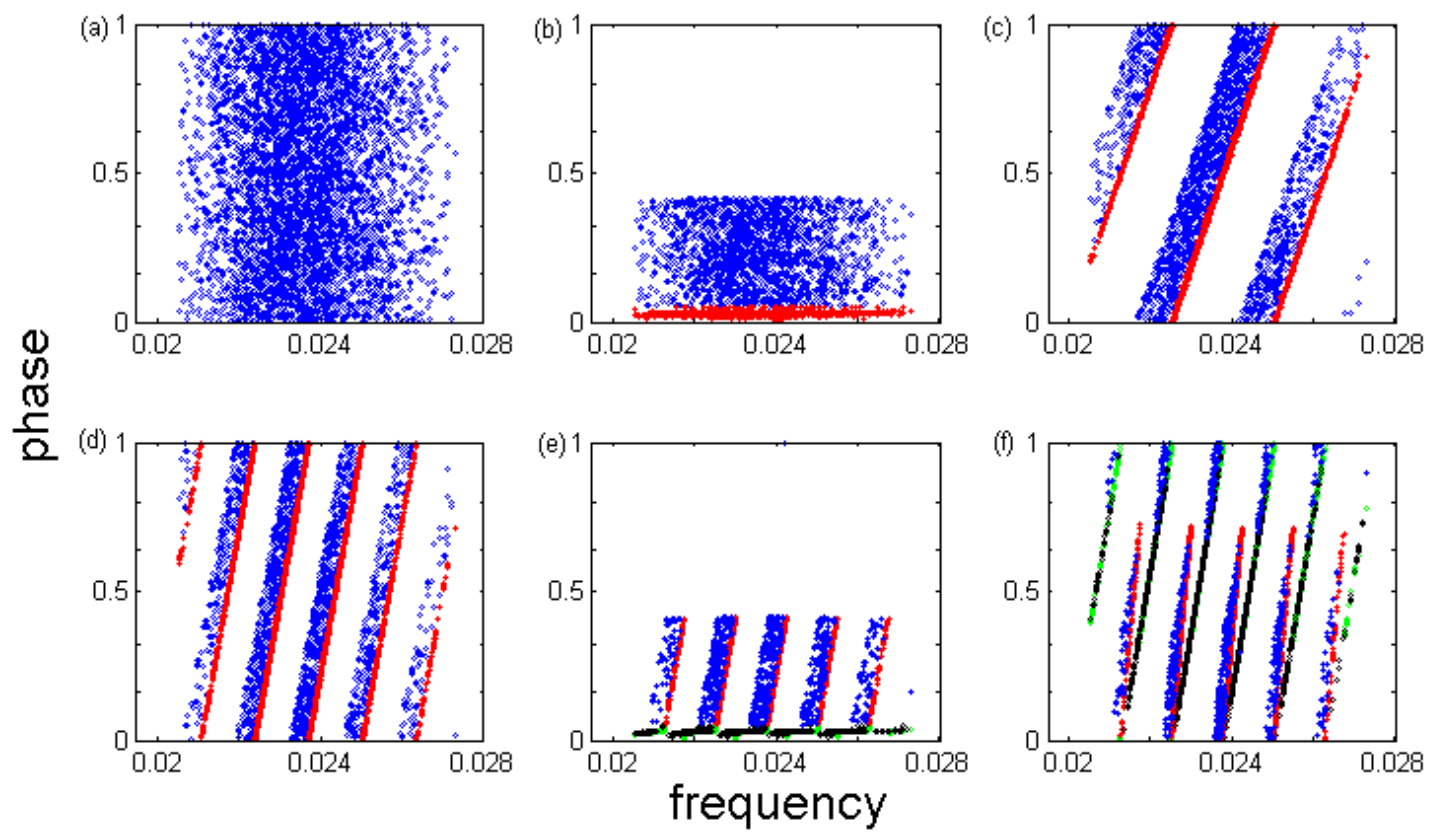

Figure 3.6: Plots of phase as a function of frequency of oscillators in the ZBKE simulation shown in Fig. 2(a) at the following times: (a) Immediately prior to first perturbation at $t_{p}$, (b) immediately following the first perturbation, in which oscillators in the resetting region of their phase are phase reset to 0 , (c) half-way between the two perturbations, $t_{p}+\tau / 2$, (d) immediately prior to the second perturbation, (e) immediately following the second perturbation, in which oscillators in the phase range $X$ through $(1-X)$ are phase reset to 0 , and (f) at the time of the echo when a series of narrow bands, each with a single branch segment is observed. Parameters: $\tau=650$; other parameters as in Figure 3.3. 
of groups I and II, we find that much of the underlying structure of the echo phenomenon can be understood, as groups III and IV have a smaller influence on the order parameter. Due to the form of Figure 3.5(a), we can approximate $X$ as the fraction of the oscillators that are phase reset by a perturbation, assuming that the oscillators are uniformly distributed through the phase at the time of a perturbation. The numbers of oscillators in groups I and II are then estimated to be $N X^{2}$ and $N X(1-X)$, respectively, in which $N$ is the total number of oscillators.

Figure 3.7 shows phase as a function of the frequency of the oscillators belonging to group I and group II at various times during a simulation. Just prior to the first perturbation, the oscillators of both of these groups are, by definition, found between phases $(1-X)$ and 1 , Figures $3.7 \mathrm{a}(\mathrm{i}), \mathrm{b}(\mathrm{i})$. Owing to the random initial conditions used in the simulation, there is no dependence of phase on frequency. Both sets of oscillators are phase reset to zero by the first perturbation. Oscillators with a higher frequency advance faster, and their phases form a set of points initially lying along a line, the steepness of which increases with time. At any arbitrary time between perturbations, the phases of the oscillators lie along a series of offset line segments due to the wrapping of the phases (from 0 to 1 ). At the time of the second perturbation, the group II oscillators, by definition, are not phase reset, and the oscillators form line segments parallel to each other, Figure $3.7 \mathrm{~b}$ (ii). In contrast, the line segments associated with group I oscillators align in the resetting region of the phase and are phase reset to 0 by the second perturbation, Figure $3.7 a(i i)$.

At the time of the echo, group I oscillators have exactly the same phase as immediately 

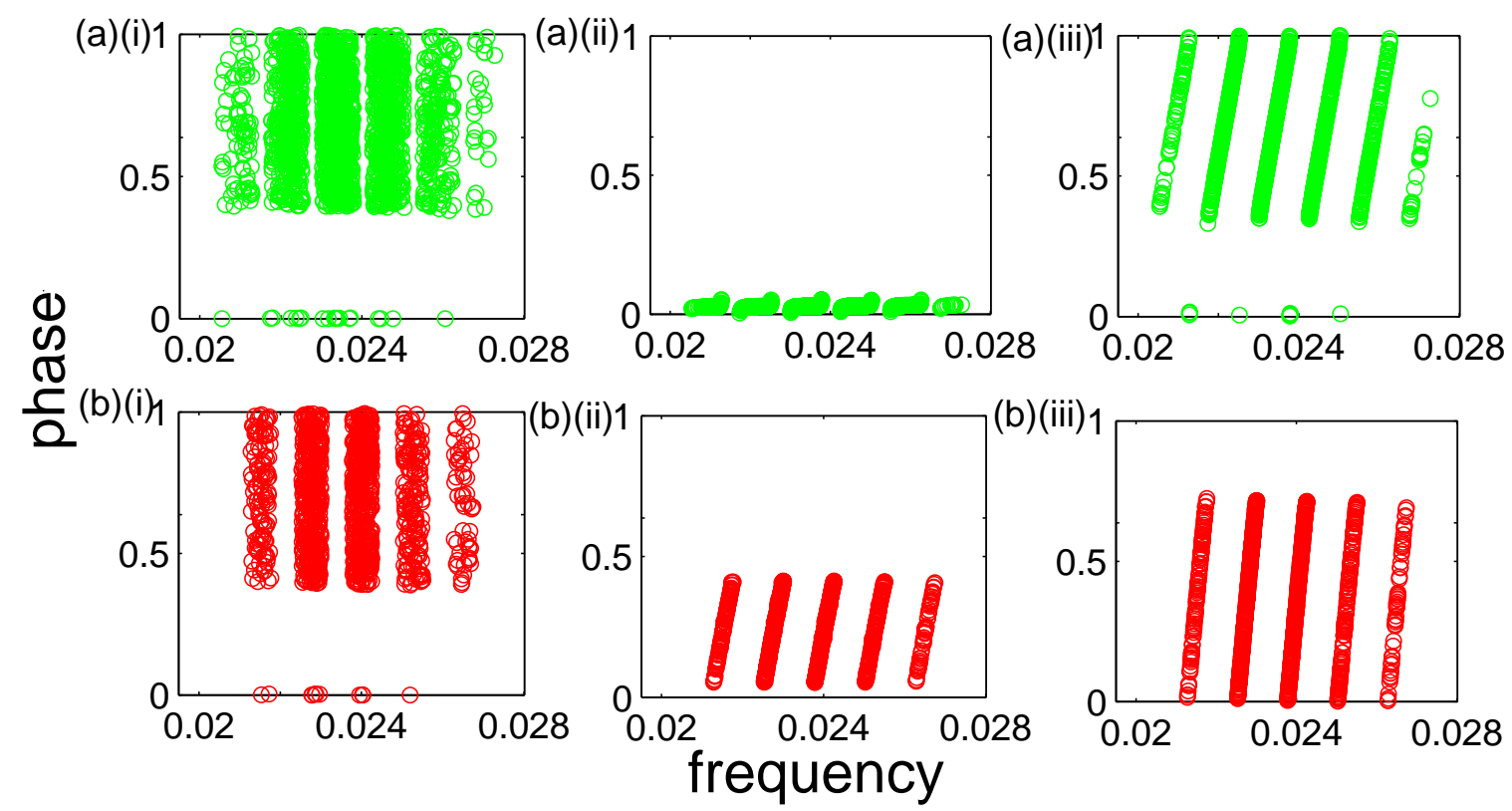

Figure 3.7: Phase as a function of the frequency of oscillators from ZBKE simulation for (a) group I oscillators and (b) group II oscillators at various times: (i) Immediately prior to the first perturbation, (ii) immediately following the second perturbation, and (iii) at the time of the echo. All parameters as in Figure 3.3.

prior to the second perturbation, with the oscillators arranged along a series of line segments mutually aligned in the phase resetting region, Figure 3.7a(iii). Group II oscillators, which were not phase reset at the second perturbation, are now aligned along a series of line segments that are twice as steep and twice as long as they were at the time of second perturbation, Figure 3.7b(iii). In Figure 3.7, there are gaps in the observed frequencies of both groups, with the missing frequencies in group I plots corresponding to the frequencies present in group II plots and vice-versa. This occurs because the definition of each group places separate restrictions on the allowed frequencies of its member oscillators. Because the 
phases of group II oscillators must lie between 0 to $(1-X)$ at time $t_{p}+\tau$ and align at zero at time $t_{p}$, the allowed frequencies of the oscillators are given by $n / \tau+\alpha / \tau$, with $\alpha$ restricted to the range $0<\alpha<(1-X)$, where $n$ can be any integer value. The separate line segments in Figure 3.7b(ii) correspond to increasing values of n, with the oscillators arranged along the central line segment having the range of frequencies corresponding to $n=15$. This is in agreement with the fact that the value of $\tau$ for this simulation is approximately 15 times the value of the mean period of the oscillators. Group I oscillators have frequencies $n / \tau+$ $\alpha / \tau$, with $\alpha$ restricted to the range $(1-X)<\alpha<1$. This ensures that they lie within the resetting region at the time of the second perturbation.

The value of the order parameter at the time of the echo can now be estimated based upon the phase distributions of group I and group II oscillators. As illustrated in Figure 3.8, group I oscillators are found between $(1-X)$ and 1 , while group II oscillators are found between 0 and $2(1-X)$. Our approach assumes that the phases of the oscillators in a given group are uniformly distributed through their respective regions, which is valid for large $N$. The actual distribution determined from a ZBKE simulation, shown in Figure 3.8(a), verifies the validity of this assumption.

The order parameter calculated using the phase distribution of group I and II oscillators as a function of $X$ is shown in Figure 3.5(a) by the light blue curve. The estimate of the order parameter can be improved further by including the impact of group III and group IV. The phase distribution of these oscillators at the time of echo is determined using a geometric approach, described in the last section. Figure 3.8(a) shows the geometrically 

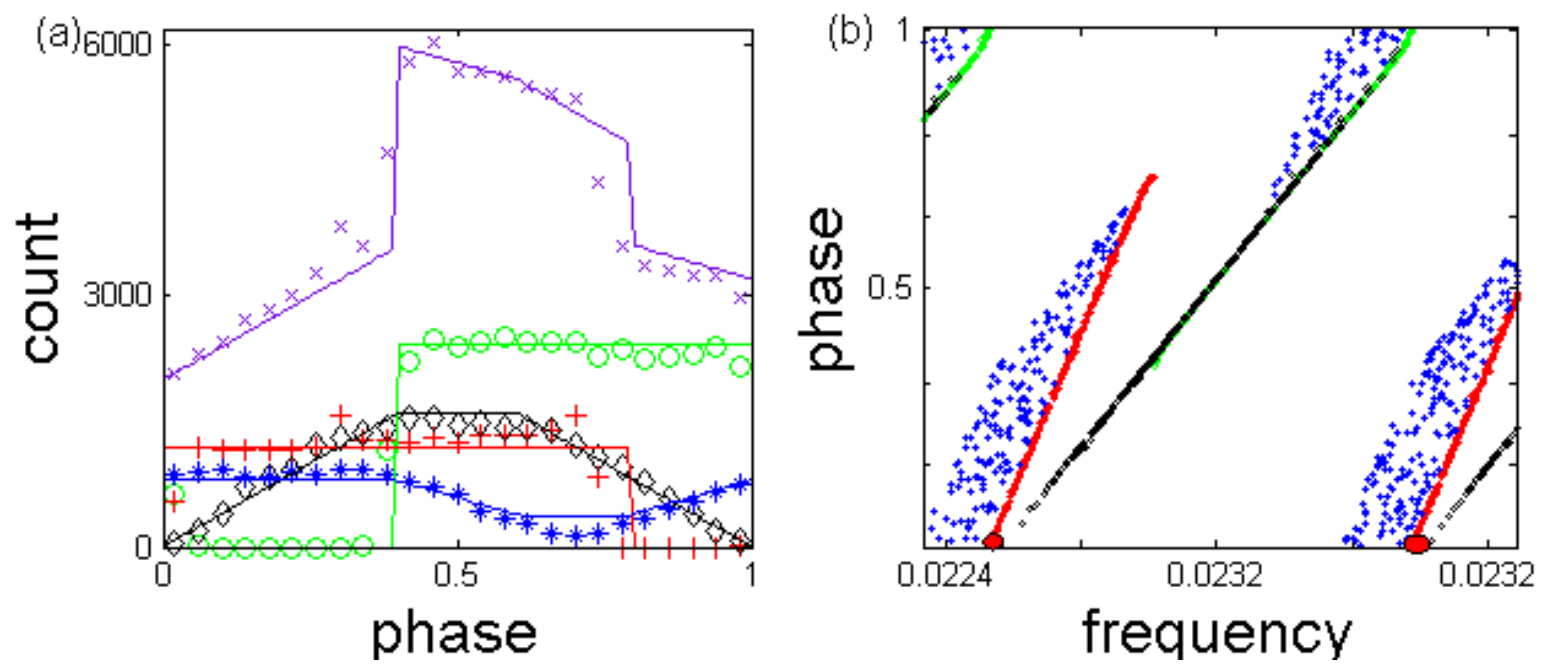

Figure 3.8: (a) Predicted phase distribution of oscillators (solid lines) and ZBKE simulation values (symbols) at the time of the echo. Green, red, black and blue lines correspond to groups I, II, III and IV, respectively. The violet line corresponds to the sum of the four groups. The ZBKE simulations were carried out with a large number of oscillators, $N=$ 100, 000. (b) Detail of structure from Figure 3.6, color coded according to the four different groups in (a) at the time of the echo. The frequencies indicated by the left and right red circles correspond to $14 / \tau$ and $15 / \tau$, respectively.

calculated distributions along with ZBKE model simulations. The overall phase distribution of all oscillators is shown in Figure 3.8(a), where the dominate asymmetry arises from the overlap of the oscillators from group I and group II, with a smaller contribution from group III and group IV. Figure 3.5(a) shows remarkably good agreement between our theoretically determined order parameter as a function of $X$ at the time of echo to that calculated using the ZBKE model.

A close-up of the central branched band seen earlier in Figure 3.6(f) is shown in Figure 
3.8(b), color coded by the four groups. The members of group I and group II are seen to span the entire range of allowed frequencies, i.e., between $14 / \tau$ and $15 / \tau$, as discussed above. Member oscillators of these two groups are uniformly distributed along their respective line segments. Group III oscillators are also distributed along a thin line, although the distribution is now not uniform. Group IV oscillators are restricted to a certain region of this phase frequency plot, which forms a band structure instead of a line, since they have never been reset by a perturbation. While only group I is affected by both perturbations, membership of any group places restrictions on an oscillator in term of natural frequency. Further, as observed in the rich structure revealed in Figure 3.8(b), each group of oscillators collectively holds the memory of this original information. The echo phenomenon, while remarkable, does not in itself reveal all of this underlying information. Only a component of the information is interrogated by calculation of the order parameter, with the increase in $R$ close to the echo time due to the alignment that occurs between the line segments associated with group I and those with group II.

\subsection{Geometric Approach}

We can use a geometric approach to determine the phase distributions of the oscillators associated with group III and group IV at the time of the echo. Consider the group III oscillators. At time $t_{p}$, these oscillators are uniformly distributed between 0 and $(1-X)$, Figure 3.9(a). At time $\tau$ later, they are uniformly distributed through the region $(1-X)$ 

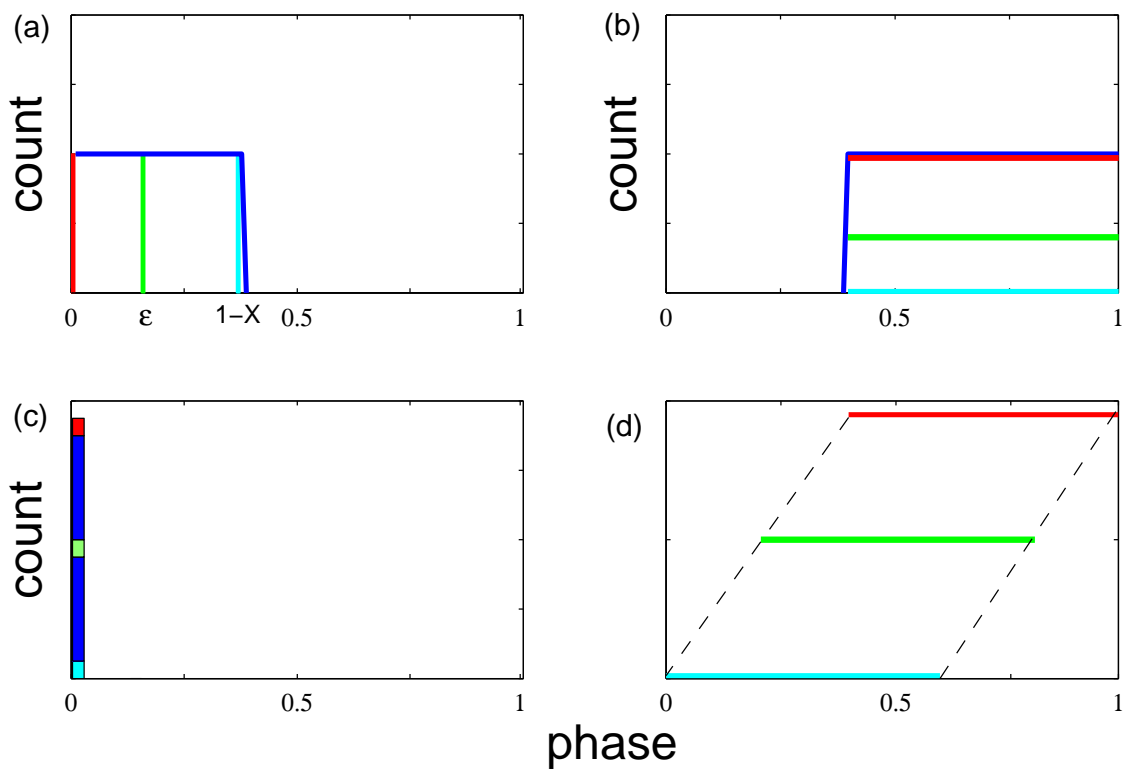

Figure 3.9: Geometric determination of phase distribution of group III oscillators. (a) At time $t_{p}$, they are uniformly distributed through the refractory region. (b) Just prior to the second perturbation, the same oscillators are now uniformly distributed through the excitable region. (c) The oscillators are then phase reset by the perturbation. The red, light blue and green regions indicate the oscillators in one of three narrow phase ranges at time $t_{p}$. The order of their vertical stacking in (b) and (c) is arbitrary. Each horizontal band equally contributes to the total number of oscillators at a particular phase. (d) The phase range of each set, red, blue and green, of oscillators at the time of the echo. Since the choice of $\epsilon$ was arbitrary, the phase spread of the other oscillators is bound by the sides of the parallelogram, black dotted line. 
to 1. This places restrictions on their allowed frequencies, though, in contrast to group I and II, the restrictions are now dependent on the particular phase of an oscillator at time $t_{p}$. Consider oscillators with the narrow range of phases indicated in red in Figure 3.9(a). At time $\tau$ later these oscillators will lie uniformly between $(1-X)$ and 1 . Therefore, the allowed frequencies of oscillators in this thin region are $n / \tau+\alpha / \tau$, with $\alpha$ restricted to the range $1-X$ through 1 . In Figure 3.9(b), we schematically draw the phase location of these oscillators, spread uniformly through the excitable region. Oscillators in the narrow range of phase indicated in light blue in Figure 3.9(a) show phase a phase advance of between 0 and $X$. In Figure 3.9(b), these oscillators are schematically illustrated as spread through the excitable region at the time of the second perturbation. Their allowed frequencies are given by $n / \tau+\alpha / \tau$, with $\alpha$ restricted to the range 0 through $X$. For the general narrow region of oscillators at phase $\epsilon$ at time $t_{p}$, indicated in green in Figure 3.9(a), (b), the value of $\alpha$ is restricted to the range $1-X-\epsilon<\alpha<1-\epsilon$. At time $t_{p}+\tau$, all of the oscillators are phase reset to 0, Figure 3.9(c). The location of any oscillator $\tau$ later can be determined using the above allowed frequencies. The oscillators that were in the red region, will advance through $(1-X)$ to 1 , whereas the oscillators that were in the light blue region will advance from 0 through to $X$. The location of each of these sets of oscillators in phase at the time of the echo are shown in Figure 3.9(d), with the red and blue oscillators off set from each other in phase. These sets of oscillators can be thought of as forming the top and bottom sides of a parallelogram. The location of an arbitrary set of oscillators, which had phases close to $\epsilon$ at time $t_{p}$, is bound by the parallelogram's other sides. The total number of 
oscillators at a particular phase, in Figure 3.9(d), is proportional to the internal height of the parallelogram at that phase. By internal height, we mean either the vertical distance from the bottom/top side to its adjacent side or the parallelogram's altitude. Assuming the altitude of the parallelogram is one, the internal height as a function of phase, $\psi$, is equal to $\psi /(1-X)$ for $0<\psi<(1-X), 1$ for $(1-X)<\psi<X$ and $(1-\psi) /(1-X)$ for $\psi>X$. This is plotted in Figure 3.8(a) as the red line.

\subsection{Conclusion}

The occurrence of an echo in this system of chemical oscillators is a counter-intuitive phenomenon. The apparent loss of information, measured using the order parameter, through inherent phase dispersion belies the fact that the information remains encoded within the phase structure of the oscillators. It is only due to the mutual alignment at the time of the echo that this encoded structure is revealed. Our method of analysis clearly shows this structure embedded within each sub-community of oscillators, Figure 3.8. Noise can lead to the destruction of the relative phase structure of the oscillators. Our simulation shows that for a given $\tau$, the size of echo decreases as the magnitude of noise in the system increases. At the sufficiently large magnitude of noise, the echo no longer occurs. Equivalently, Ott el al. showed that for fixed magnitude of noise, the size of the echo decreases with increasing delay value $\tau$ [14]. The observation of an echo in our experimental systems, Figure 3.1, indicates, therefore, that the experimental system is within the range where the time-integrated 
amount of noise is sufficiently small that the encoded phase structure is not destroyed, Figure 3.2. Within this range, as within our numerical simulations subject to small amounts of noise, the echo phenomenon is robust.

The present work serves as the first example of echo phenomena in a community of chemical oscillators and supports the finding of previous theoretical work based upon phase oscillators [14]. It demonstrates that the echo phenomenon is robust enough to occur in a real-world system. Further, it suggests that the echo phenomenon could be observed and/or exploited in other natural oscillatory systems representable using a phase model approach. 


\section{Bibliography}

[1] K. Agladze and V. Krinsky. "Multi-armed vortices in an active chemical medium". Nat., 296(5856):424-426, 1982.

[2] J. Aldridge and E. K. Pye. "Cell density dependence of oscillatory metabolism". Nat., 259(5545):670-671, 1976.

[3] G. Buzsáki and A. Draguhn. "Neuronal oscillations in cortical networks". Sci., 304(5679):1926-1929, 2004.

[4] D. Dormann, B. Vasiev, and C. J. Weijer. "Propagating waves control Dictyostelium discoideum morphogenesis". Biophys. Chem., 72(1):21-35, 1998.

[5] L. Glass. "Synchronization and rhythmic processes in physiology". Nat., 410(6825):277-284, 2001.

[6] R. W. Gould. "Cyclotron Echo Phenomena". Am. J. Phys., 37(6):585, 1969.

[7] E. L. Hahn. "Spin echoes". Phys. Rev., 80(4):580, 1950. 
[8] V. Horvath, P. L. Gentili, V. K. Vanag, and I. R. Epstein. "Pulse-coupled chemical oscillators with time delay". Angewandte Chemie Int. Ed., 51(28):6878-6881, 2012.

[9] H. Ke, M. R. Tinsley, A. Steele, F. Wang, and K. Showalter. "Link weight evolution in a network of coupled chemical oscillators". Phys. Rev. E, 89(5):052712, 2014.

[10] Y. Kuramoto. Chemical Oscillations, Waves, and Turbulence. Springer Science \& Business Media, 2012.

[11] J. Malmberg, C. Wharton, R. Gould, and T. O'Neil. "Plasma wave echo experiment". Phys. Rev. Lett., 20(3):95, 1968.

[12] H. Nakao, K. Arai, and Y. Kawamura. "Noise-induced synchronization and clustering in ensembles of uncoupled limit-cycle oscillators". Phys. Rev. Lett., 98(18):184101, 2007.

[13] S. Nkomo, M. R. Tinsley, and K. Showalter. "Chimera states in populations of nonlocally coupled chemical oscillators". Phys. Rev. Lett., 110(24):244102, 2013.

[14] E. Ott, J. H. Platig, T. M. Antonsen, and M. Girvan. "Echo phenomena in large systems of coupled oscillators". Chaos, 18(3):037115, 2008.

[15] H. M. Smith. "Synchronous flashing of fireflies". Sci., 82(2120):151-152, 1935. 
[16] A. F. Taylor, P. Kapetanopoulos, B. J. Whitaker, R. Toth, L. Bull, and M. R. Tinsley. "Clusters and switchers in globally coupled photochemical oscillators". Phys. Rev. Lett., $\mathbf{1 0 0}(21): 214101,2008$.

[17] A. F. Taylor, M. R. Tinsley, F. Wang, and K. Showalter. "Phase clusters in large populations of chemical oscillators". Angewandte Chemie, 123(43):10343-10346, 2011.

[18] M. R. Tinsley, S. Nkomo, and K. Showalter. "Chimera and phase-cluster states in populations of coupled chemical oscillators". Nat. Phys., 8(9):662-665, 2012.

[19] M. R. Tinsley, A. F. Taylor, Z. Huang, F. Wang, and K. Showalter. "Dynamical quorum sensing and synchronization in collections of excitable and oscillatory catalytic particles". Phys. D: Nonlinear Phenom., 239(11):785-790, 2010.

[20] J. F. Totz, R. Snari, D. Yengi, M. R. Tinsley, H. Engel, and K. Showalter. "Phaselag synchronization in networks of coupled chemical oscillators". Phys. Rev. E, 92(2):022819, 2015.

[21] A. N. Zaikin and A. M. Zhabotinsky. "Concentration wave propagation in twodimensional liquid-phase self-oscillating system". Nat., 225(5232):535-537, 1970.

[22] A. M. Zhabotinsky, F. Buchholtz, A. B. Kiyatkin, and I. R. Epstein. "Oscillations and waves in metal-ion-catalysed bromate oscillating reactions in highly oxidized states". J. Phys. Chem., 97(29):7578-7584, 1993. 


\title{
Echo Phenomena in Populations of Chemical Oscillators
}

\author{
Tianran Chen
}

\section{THESIS}

Submitted to the Eberly College of Arts and Sciences at

West Virginia University

in Partial Fulfillment of the Requirements for

- the Degree of

Master of Science

in

Chemistry

APPROVAL OF THE EXAMINING COMMITTEE
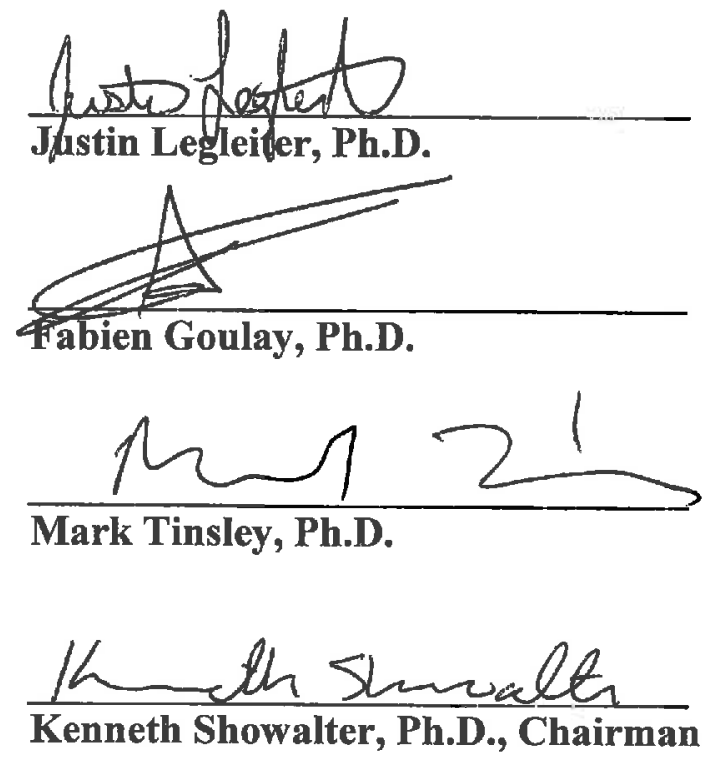

$\frac{6 / 21 / 20 / 6}{\text { Date }}$ 